\title{
The Role of Information Technology Department against the Hook Style Energy Theft in Smart Cities - Ad-Hoc Overhead Low-Voltage Broadband over Power Lines (OV LV BPL) Networks
}

\author{
Athanasios G. Lazaropoulos* \\ School of Electrical and Computer Engineering / National Technical University of Athens / \\ 9 Iroon Polytechniou Street / Zografou, GR 15780
}

Received February 24, 2019; Accepted March 26, 2019; Published April 2, 2019

\begin{abstract}
This paper investigates the possibility of detecting the hook style energy theft in the overhead low-voltage (OV LV) power grids through the hook style energy theft method (HS-DET method) when ad-hoc overhead Low-Voltage Broadband over Power Lines (OV LV BPL) networks are deployed by the Information Technology departments of the power utilities. Without the need for the deployment of a complete and permanent OV LV BPL network across the OV LV power grid, the impact of the deviation from the initial measurement positions and of longer ad-hoc OV LV BPL topologies on the detection efficiency of HS-DET method is assessed by using the already validated percent error sum (PES) metrics and appropriate contour plots.
\end{abstract}

Keywords: Smart Grid; Broadband over Power Lines (BPL); Power Line Communications (PLC); Distribution Power Grid; Energy Theft; Jamming; Robustness

\section{Introduction}

Energy theft defines a well-known problem either in traditional power systems or in the emerging smart grid while the financial losses of the power utilities reach up to $\$ 6$ billion, $\$ 5$ billion and $\$ 4.5$ billion in United States, Brazil and India, respectively [1]-[10]. The existing portfolio of BPL broadband applications, such as Topology Identification Methodology (TIM) [11], Fault and Instability Identification Methodology (FIIM) [12], methodology to preserve power system stability [13]-[16] and Main Line Fault Localization Methodology (MLFLM) [17]-[19], that has been recently enriched with the hook style energy theft detection method (HS-DET method) [20]-[22] can monitor, meter, control and provide valuable real-time detailed information on actual operation of the power grid while it can identify and stop fraud (energy theft).

Until now [20]-[22], HS-DET method, which is based on the hybrid model [23]-[40], has been assumed to operate on the basis of a permanently installed BPL network while examining the permanent BPL cascaded topologies of this BPL network. In fact, HS-DET method adopts $\triangle P E S$ metric, which is the suitable percent error sum (PES) submetric for energy theft detection, and its relevant contour plots in order to detect the hook style energy theft across the OV LV BPL topologies [20]-[22]. 
In this paper, to avoid the cost and the delay of a permanent installation of a BPL network, Information Technology departments of the power utilities can deploy ad-hoc OV LV BPL topologies so that an energy theft can be detected.

Actually, $\triangle P E S$ and its relevant contour plots are going to be again exploited during the energy theft detection of ad-hoc OV LV BPL topologies since they are going to evaluate the asymmetry between the original and the modified ad-hoc OV LV BPL topology where the modified ad-hoc OV LV BPL topology comes from the original adhoc OV LV BPL topology after the hook insertion. However, the asymmetry evaluation implies that the Information Technology departments have already deployed the same adhoc OV LV BPL topologies during the normal operation of the power grid so that available measurements are available.

HS-DET method has first been presented in [20] where the impact of OV LV BPL topologies, hook characteristics and measurement differences on the performance of HS-DET method has been assessed through $\overline{\triangle P E S}$ metric and its relevant contour plots. In [21], a first set of special issues, such as: (i) the existence / definition of a threshold of the intensity of measurement differences that may occur / be produced preventing faulty decisions concerning the existence of hook style energy theft; (ii) the impact of the installation of very long hooks; and (iii) the impact of the use of "smart" hooks, has been examined. In [22], a second set of special issues, such as: (a) the impact of different CUD measurement differences of the same maximum value when the coupling transfer functions of the original and the modified OV LV BPL topologies are measured; (b) the installation of two "smart" hooks; and (c) the impact of the full interconnection assumption of the "smart" hook during the computations of HS-DET method, has also been addressed. Taking into consideration the findings of [20]-[22] and the special nature of ad-hoc OV LV BPL topologies, a set of following issues related to the operation of ad-hoc OV LV BPL topologies is investigated in this paper, namely: (i) the effect of the deviation from the initial measurement positions of the installed ad-hoc OV LV BPL topology by the Information Technology department; and (ii) the effect of application of longer ad-hoc OV LV BPL topologies than the typical OV LV BPL topologies of the permanent BPL networks. The impact of the aforementioned two special features of the ad-hoc OV LV BPL topologies on the performance of the HS-DET method is assessed by using the already validated $\triangle P E S$ and its appropriate contour plots.

The rest of this paper is organized as follows: In Section 2, a presentation of the proposed ad-hoc OV LV BPL topology installation concept is given as well as the required assumptions concerning the operation of ad-hoc OV LV BPL topologies. In Section 3, the synopsis of HS-DET method and PES metrics is presented. In Section 4, numerical results and discussion are provided, aiming at practically evaluating the performance of HS-DET method when ad-hoc OV LV BPL topologies are deployed.

\section{Ad-hoc OV LV BPL Topologies}

Ad-hoc OV LV BPL topologies are deployed upon the OV LV power grid resembling to the cascaded OV LV BPL topologies of the permanent BPL networks. A typical OV LV power grid consists of OV LV distribution power lines such as this of Fig. 1 of [20]. The dimensions of the OV LV multiconductor transmission line (MTL) configuration, the characteristics of the involved imperfect ground and the properties of 
the BPL signal propagation / transmission through OV LV MTL configurations are detailed in [23], [25]-[29], [31]-[33], [41]-[46].

In the case of a permanent and complete BPL network installation, a BPL network can be divided into cascaded BPL topologies while in the case of the ad-hoc BPL topologies, which are studied in this paper, the ad-hoc BPL topology is synonym to the BPL network. In Fig. 1(a), a typical ad-hoc BPL topology with $N$ branches, which does not differentiate from a typical BPL topology of a permanent BPL network, is shown. With respect to Fig. 1(a), an ad-hoc BPL topology is bounded by its transmitting and receiving ends. BPL units that operate as the transmitting and receiving ends are treated as the network gateways of the Information Technology department for its measurements. The number of branches and the topological characteristics of ad-hoc BPL topologies vary depending on the location of the examined segment of the OV LV power grid; say, this location defines the OV LV BPL topology class. In Table 1, the topological characteristics of indicative ad-hoc OV LV BPL topologies are reported. Note that same indicative ad-hoc OV LV BPL topologies with the indicative OV LV BPL topologies of typical lengths $D=\sum_{i=1}^{N+1}\left(L_{i}\right)$ of $1000 \mathrm{~m}$ are initially assumed in this paper (see [20]).

During the normal operation of the power grid, the Information Technology department should collect channel attenuation measurements from the examined ad-hoc OV LV BPL topology that is hereafter denoted as the original ad-hoc OV LV BPL topology. These measurements will also act as the reference measurements of the HS-DET method and should be properly handled by a database management system. Now, with reference to Fig. 1(b), let assume that a hook style energy theft occurs at the position $A_{h}$ that is located at distance $D_{\mathrm{h}}=L_{\mathrm{h}}+\sum_{i=1}^{k-1}\left(L_{i}\right)$ from the transmitting end. The hook is located at distance $L_{k}-L_{\mathrm{h}}$. from the $k$ th branch while its length is equal to $L_{\text {bh. }}$ In total, the modified ad-hoc OV LV BPL topology is illustrated in Fig. 1(b) with reference to the original ad-hoc OV LV BPL topology of Fig. 1(a).

Apart from the topological characteristics of Table 1, a set of assumptions concerning the transmission and propagation of the BPL signal as well as the circuital characteristics of the OV LV MTL configurations, which are required to simplify the following analysis, are detailed in [23]-[28]. Except for the aforementioned assumptions, assumptions concerning the hook handling should be made, namely: (i) the hook termination is assumed to be open circuit; and (ii) the hook interconnection with the distribution lines is assumed to be complete (i.e., hook derivation points at the same distance from the transmitting end on all the three phases) and horizontal. Apart from the previous hook handling assumptions, no measurement differences are assumed in this paper. Although differentiations of the latter assumptions have been thoroughly examined and assessed in terms of the performance of the HS-DET method in [20]-[22], their simplest versions are assumed in this paper for the sake of the paper size reduction.

\section{HS-DET Method Synopsis and the Ad-Hoc OV LV BPL Topology Case}

\subsection{Hybrid Model and BPL Signal Coupling}

As the channel attenuation measurements of the Information Technology department are concerned, the hybrid model, which describes the BPL propagation and transmission, is here applied. Extensively analyzed in [23]-[29], [32], 
hybrid model consists of two interconnected modules, say, the bottom-up and the top-down approach module, while several useful spectral efficiency metrics such as channel attenuation and capacity may be calculated. In accordance with the bottom-up approach of the hybrid model, the MTL configuration of the examined ad-hoc OV LV BPL topologies may support 4 modes that propagate across the MTL BPL configuration.

a)

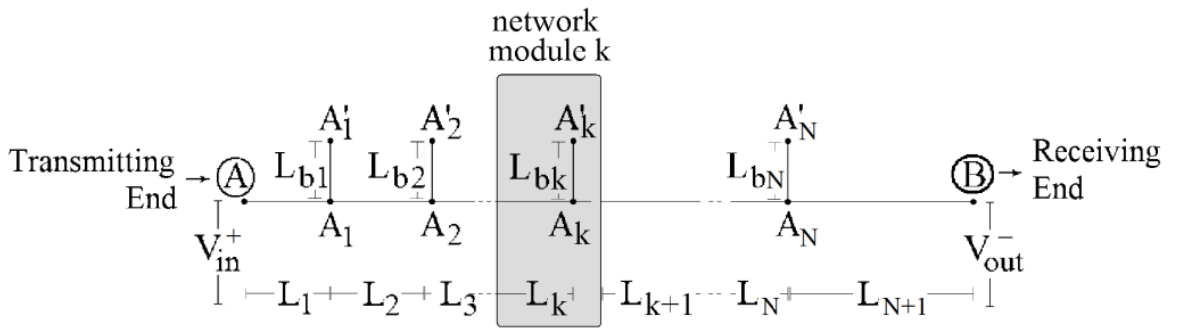

b)

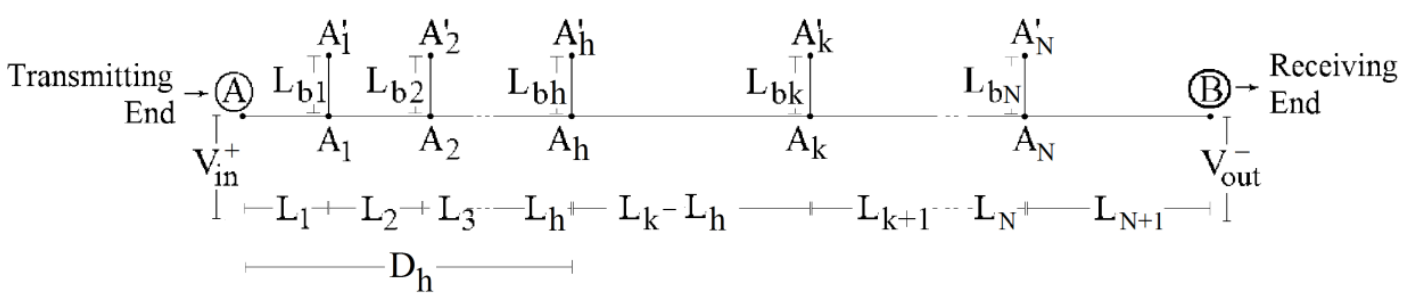

Fig. 1. (a) Typical original ad-hoc OV LV BPL topology with $N$ branches [20]. (b) Typical modified ad-hoc OV LV BPL topology due to the hook insertion.

Table 1

Indicative Original Ad-Hoc OV LV BPL Topologies [20]

\begin{tabular}{|c|c|c|c|c|}
\hline $\begin{array}{c}\text { Topology } \\
\text { Name } \\
\text { (OV LV BPL } \\
\text { Topology } \\
\text { Class) }\end{array}$ & Topology Description & $\begin{array}{c}\text { Number of } \\
\text { Branches }\end{array}$ & $\begin{array}{c}\text { Length of } \\
\text { Distribution Lines }\end{array}$ & $\begin{array}{c}\text { Length of } \\
\text { Branching Lines }\end{array}$ \\
\hline Urban case A & Typical OV urban topology & 3 & $\begin{array}{c}L_{1}=500 \mathrm{~m}, L_{2}=200 \mathrm{~m}, \\
L_{3}=100 \mathrm{~m}, L_{4}=200 \mathrm{~m}\end{array}$ & $\begin{array}{c}L_{\mathrm{b} 1}=8 \mathrm{~m}, L_{\mathrm{b} 2}=13 \mathrm{~m}, \\
L_{\mathrm{b} 3}=10 \mathrm{~m}\end{array}$ \\
\hline Urban case B & $\begin{array}{c}\text { Aggravated OV urban } \\
\text { topology }\end{array}$ & 5 & $\begin{array}{c}L_{1}=200 \mathrm{~m}, L_{2}=50 \mathrm{~m}, \\
L_{3}=100 \mathrm{~m}, L_{4}=200 \mathrm{~m}, \\
L_{5}=300 \mathrm{~m}, L_{6}=150 \mathrm{~m}\end{array}$ & $\begin{array}{c}L_{\mathrm{b} 1}=12 \mathrm{~m}, L_{\mathrm{b} 2}=5 \mathrm{~m}, \\
L_{\mathrm{b}}=28 \mathrm{~m}, L_{\mathrm{b} 4}=41 \mathrm{~m}, \\
L_{\mathrm{b} 5}=17 \mathrm{~m}\end{array}$ \\
\hline Suburban case & OV suburban topology & 2 & $L_{1}=500 \mathrm{~m}, L_{2}=400 \mathrm{~m}$, \\
$L_{3}=100 \mathrm{~m}$ & $L_{\mathrm{b} 1}=50 \mathrm{~m}, L_{\mathrm{b} 2}=10 \mathrm{~m}$ \\
\hline Rural case & OV rural topology & 1 & $L_{1}=600 \mathrm{~m}, L_{2}=400 \mathrm{~m}$ & $L_{\mathrm{b} 1=300 \mathrm{~m}}$ \\
\hline "LOS" case & $\begin{array}{c}\text { OV Line-of-Sight } \\
\text { transmission }\end{array}$ & 0 & $L_{1}=1000 \mathrm{~m}$ & - \\
\hline
\end{tabular}

With reference to Figs. 1(a) and 1(b), through the combined operation of TM2 method, which is based on the scattering matrix formalism and is part of the top-down approach of the hybrid model [47], and CS2 module, which deals with the way that the BPL signal is injected onto and extracted from the OV LV lines and is also part of the top-down approach of the hybrid model [48], [49], the coupling scheme channel transfer function 
that relates output BPL signal of the receiving end and input BPL signal of the transmitting end is given by

$$
H^{\mathrm{c}}\{\boldsymbol{*}\}=\left[\mathbf{C}^{\text {out }}\right]^{\mathrm{C}} \cdot\left[\mathbf{T}_{\mathrm{V}} \times \mathbf{H}^{\mathrm{m}}\{\mathrm{x}\} \cdot\left(\mathbf{T}_{\mathrm{V}}\right)^{-1}\right] \cdot\left[\mathbf{C}^{\text {in }}\right]^{\mathrm{C}}
$$

where $\mathbf{H}^{\mathrm{m}}\{x\}$ is the $4 \times 4$ modal channel transfer function matrix of the examined OV LV MTL configuration, $\mathbf{T}_{\mathrm{V}}^{\mathrm{G}}$ is a $4 \times 4$ matrix depending on the frequency, the examined distribution power grid type, the physical properties of the cables and the geometry of the MTL configuration, $\mathbf{C}^{\text {in }}$ is the $1 \times 4$ input coupling vector, $\mathrm{C}^{\text {out }}$ is the $4 \times 1$ output coupling vector and $\left.{ }^{\left[{ }^{\circ}\right.}\right]^{\mathrm{C}}$ denotes the applied coupling scheme. In accordance with [48], [49], CS2 module may support three types of coupling schemes in ad-hoc OV LV BPL topologies, namely: (i) Coupling Scheme Type 1: Wire-to-Ground (WtG) coupling schemes; (ii) Coupling Scheme Type 2: Wire-to-Wire (WtW) coupling schemes, and (iii) Coupling Scheme Type 3: MultiWire-to-MultiWire (MtM) coupling schemes. Depending on the applied coupling scheme, $\mathbf{C}^{\text {in }}$ and $\mathbf{C}^{\text {out }}$ are properly defined, as dictated in [48], [49], and, then, the coupling scheme channel transfer function $H^{\mathrm{c}}\{\mathrm{x}\}$ is straightforward computed from eq. (1).

\subsection{HS-DET Method}

With reference to eq.(1), [20] and [21], to apply HS-DET method when no measurement differences are assumed and the original ad-hoc OV LV BPL topologies are considered, the original measured coupling scheme transfer function $\overline{H_{\mathrm{nr}}^{\mathrm{C}}}\{x\}$, which is measured by the Information Technology department, for given coupling scheme can be determined by

$$
\begin{gathered}
\overline{H_{\mathrm{nr}}^{\mathrm{C}}}\left(f_{i}\right)=H_{\mathrm{mr}}^{\mathrm{c}}\left(f_{i}\right)+e\left(f_{i}\right), i=1, \ldots, u \\
e\left(f_{i}\right)=0, i=1, \ldots, u
\end{gathered}
$$

where $f_{i}, i=1, \ldots, u$ denotes the measurement frequency, $u$ is the number of subchannels in the examined frequency range and $e\left(f_{i}\right)$ synopsizes the total measurement difference in $\mathrm{dB}$ at frequency $f_{i}$. that is anyway equal to zero in this paper.

As already mentioned, each hook can be treated as an open circuit fully interconnected horizontal branch by the hybrid model while the hook insertion modifies the original ad-hoc OV LV BPL topology to the respective modified one. With reference to eqs. (2) and (3), $\overline{H_{\text {mond }}^{\mathrm{C}}}\{x\}$ can be considered as the modified measured coupling scheme channel transfer function, which is measured by the Information Technology department, that anyway coincides with the modified theoretical coupling scheme channel transfer function $H_{\bmod }^{\mathrm{C}}\{x\}$ due to the no measurement difference assumption of this paper.

Apart from the channel attenuation measurements of the Information Technology department, its main concern is the computation of PES submetrics that are the cornerstone metrics of HS-DET method and define whether a hook style energy theft occurs or not. In accordance with [20], the PES submetrics that are used in this paper when the no measurement difference assumption occurs are:

$$
\begin{gathered}
P E S_{\text {or }}=100 \% \times \frac{\sum_{i=1}^{u}\left|H_{0 r}^{C}\left(f_{i}\right)-H_{0 r}^{C}\left(f_{i}\right)\right|}{\sum_{i=1}^{u}\left|H_{0 r}^{C}\left(f_{i}\right)\right|}=0 \% \\
P E S_{\bmod }=100 \% \times \frac{\sum_{i=1}^{u}\left|H_{\bmod }^{C}\left(f_{i}\right)-H_{0 r}^{C}\left(f_{i}\right)\right|}{\sum_{i=1}^{u}\left|H_{0 r}^{C}\left(f_{i}\right)\right|} \\
\triangle P E S=P E S_{\bmod }-P E S_{\text {or }}=P E S_{\bmod }
\end{gathered}
$$


where $P E S_{\text {or }}$ and $P E S_{\text {mod }}$ assess the accuracy of the original measured coupling scheme channel transfer function and modified measured coupling scheme channel transfer functions, respectively, in relation with the original theoretical coupling scheme channel transfer function while $\triangle P E S$ is the difference between the original and modified measured coupling scheme channel transfer functions. Based on $\triangle P E S$ computations of eq. (6), appropriate contour plots may be plotted that facilitate the hook style energy theft detection [20]-[22]. In accordance with [21], suitable $\overline{\triangle P E S}$ thresholds have been proposed towards the safe hook style energy theft detection; HS-DET method can detect the energy theft through its strict $\overline{\triangle P E S}$ threshold of $10 \%$ in the vast majority of the OV LV BPL topologies while the loose $\overline{\triangle P E S}$ threshold of HS-DET method of $0 \%$ allows the energy theft in the remaining OV LV BPL topologies (e.g., urban OV LV BPL topologies when very high measurement differences occur) with the risk of a false alarm. In this paper, the same conclusions regarding the $\overline{\triangle P E S}$ thresholds of [21] are applied for the $\triangle P E S$ computations.

A number of different scenarios concerning the operation of the HS-DET method has been examined until now while two critical but special issues concerning the operation of the ad-hoc OV LV BPL topologies are here outlined and investigated. Until now, the impact of OV LV BPL topologies, hook characteristics and measurement differences on the performance of HS-DET method has been assessed in [20] through $\overline{\triangle P E S}$ metric and its relevant contour plots. In [21], apart from the decision $\overline{\triangle P E S}$ threshold regarding the energy theft detection, the impact of the installation of very long hooks and the use of "smart" hooks has been examined. In [22], the impact of different CUD measurement differences, the installation of two "smart" hooks and the full interconnection assumption has also been assessed. Taking into consideration the findings of [20]-[22], two issues related to the operation of ad-hoc OV LV BPL topologies are investigated in this paper, namely:

- The effect of the deviation from the initial measurement positions. As already mentioned, in order to detect a hook style energy theft, Information Technology department should have already installed the original ad-hoc OV LV BPL topology and should have gathered reference measurements for the topologies of interest (e.g., the indicative ad-hoc OV LV BPL topologies of Table 1) during the normal operation of the OV LV power grid. Therefore, the transmitting and receiving ends, which define the initial measurement positions, are well defined while the length $D$ between the ends is known (e.g., the typical length of the indicative ad-hoc OV LV BPL topologies of Table 1 is equal to $1000 \mathrm{~m}$ ). With reference to eq. (6), when an energy theft is suspected, Information Technology department should again install the ad-hoc OV LV BPL topology at the same measurement positions with the original ones. But, small measurement location deviations $d e v_{\mathrm{t}}$ and $d e v_{\mathrm{r}}$ may occur at the transmitting and receiving end, respectively, due to either position miscalculations of the Information Technology department or physical reasons having to do with the OV LV power grid. In Table 2, the respective modified ad-hoc OV LV BPL topologies of ones of Table 1 without the hook insertion are reported when the measurement location deviations $d e v_{\mathrm{t}}$ and $d e v_{\mathrm{r}}$ may take positive or negative values (the meaning of a positive or negative measurement location deviation is explained in Sec. 4.1). Two scenarios concerning the measurement location deviations $d e v_{\mathrm{t}}$ and $d e v_{\mathrm{r}}$ can occur and are examined in this paper: 
- Scenario A: In this first scenario, the length $D$ of the modified ad-hoc OV LV BPL topology without the hook remains the same with the original one. Since the length between the two aforementioned BPL topologies remains the same, the measurement location deviations $d e v_{\mathrm{t}}$ and $d e v_{\mathrm{r}}$ are related according to:

$$
d e v_{\mathrm{r}}=-d e v_{\mathrm{t}}
$$

Table 2

Modified Ad-Hoc OV LV BPL Topologies without Hook Style Energy Theft with Respect to the Measurement Location Deviation

\begin{tabular}{|c|c|c|c|c|}
\hline $\begin{array}{c}\text { Topology } \\
\text { Name } \\
\text { (OV LV BPL } \\
\text { Topology } \\
\text { Class) }\end{array}$ & Topology Description & $\begin{array}{c}\text { Number } \\
\text { of } \\
\text { Branches }\end{array}$ & $\begin{array}{c}\text { Length of } \\
\text { Distribution Lines }\end{array}$ & $\begin{array}{c}\text { Length of } \\
\text { Branching Lines }\end{array}$ \\
\hline Urban case A & $\begin{array}{c}\text { Shifted typical OV LV } \\
\text { urban topology without } \\
\text { hook }\end{array}$ & 3 & $\begin{array}{c}L_{1}=500 \mathrm{~m}+d e v_{\mathrm{t}}, \\
L_{2}=200 \mathrm{~m}, L_{3}=100 \mathrm{~m}, \\
L_{4}=200 \mathrm{~m}+d e v_{\mathrm{r}}\end{array}$ & $\begin{array}{c}L_{\mathrm{b} 1}=8 \mathrm{~m}, L_{\mathrm{b} 2}=13 \mathrm{~m}, \\
L_{\mathrm{b} 3}=10 \mathrm{~m}\end{array}$ \\
\hline Urban case B & $\begin{array}{c}\text { Shifted aggravated OV LV } \\
\text { urban topology without } \\
\text { hook }\end{array}$ & 5 & $\begin{array}{c}L_{1}=200 \mathrm{~m}+d e v_{\mathrm{t}}, \\
L_{2}=50 \mathrm{~m}, L_{3}=100 \mathrm{~m}, \\
L_{4}=200 \mathrm{~m}, L_{5}=300 \mathrm{~m}, \\
L_{6}=150 \mathrm{~m}+d e v_{\mathrm{r}}\end{array}$ & $\begin{array}{c}L_{\mathrm{b} 1}=12 \mathrm{~m}, L_{\mathrm{b} 2}=5 \mathrm{~m}, \\
L_{\mathrm{b} 3}=28 \mathrm{~m},\end{array}$ \\
\hline Suburban case & $\begin{array}{c}\text { Shifted OV LV suburban } \\
\text { topology without hook }\end{array}$ & 2 & $\begin{array}{c}L_{1}=500 \mathrm{~m}+d e v_{\mathrm{t}}, \\
L_{2}=400 \mathrm{~m}, \\
L_{3}=100 \mathrm{~m}+d e v_{\mathrm{r}}\end{array}$ & $L_{\mathrm{b} 1}=50 \mathrm{~m}, L_{\mathrm{b} 2}=10 \mathrm{~m}$ \\
\hline Rural case & $\begin{array}{c}\text { Shifted OV LV rural } \\
\text { topology without hook }\end{array}$ & 1 & $\begin{array}{c}L_{1}=600 \mathrm{~m}+d e v_{\mathrm{t}}, \\
L_{2}=400 \mathrm{~m}+d e v_{\mathrm{r}}\end{array}$ & $L_{\mathrm{b} 1=300 \mathrm{~m}}$ \\
\hline "LOS" case & $\begin{array}{c}\text { Shifted OV LV Line-of- } \\
\text { Sight transmission without } \\
\text { hook }\end{array}$ & 0 & $L_{1}=1000 \mathrm{~m}+d e v_{\mathrm{t}}+d e v_{\mathrm{r}}$ & \\
\hline & n & & \\
\hline
\end{tabular}

- Scenario B: In this second scenario, the length $D$ of the modified ad-hoc OV LV BPL topology without the hook is different from the original one. In this general case, the measurement location deviations $d e v_{\mathrm{t}}$ and $d e v_{\mathrm{r}}$ are not related each other and can be independently modified.

With reference to Table 1, 2 and Fig. 1(b), the modified ad-hoc OV LV BPL topology after the hook insertion occurs when a hook style energy theft occurs at the position $A_{h}$ that is located at distance $D_{h}$ from the transmitting end of the original ad-hoc OV LV BPL topology. The performance of HS-DET method should be assessed for both scenarios after the hook insertion.

- The effect of longer ad-hoc OV LV BPL topologies than the typical ones. With reference to Table 1 and 2, the indicative ad-hoc OV LV BPL topologies are assumed to have the typical lengths of $1000 \mathrm{~m}$, which are the same with the lengths of the cascaded OV LV BPL topologies of permanent BPL networks. In accordance with [11]-[14], [17]-[21], [23]-[28], although this is the typical topology length during the installation of complete BPL networks, longer ad-hoc OV LV BPL topologies can be deployed since across a length of $1000 \mathrm{~m}$ a hook style energy theft can be easily detected through a simple optical 
inspection. For that reason, concatenations of the same indicative ad-hoc OV LV BPL topologies of Table 1 are assumed for the study of the effect of longer ad-hoc OV LV BPL topologies in urban, suburban and rural environments. In Table 3, the respective modified ad-hoc OV LV BPL topologies of ones of Table 1 without the hook insertion are reported

Table 3

Modified Ad-Hoc OV LV BPL Topologies without Hook Style Energy Theft with Respect to the Concatenation of Original Ad-Hoc OV LV BPL Topologies

\begin{tabular}{|c|c|c|c|c|}
\hline $\begin{array}{c}\text { Topology } \\
\text { Name } \\
\text { (OV LV BPL } \\
\text { Topology } \\
\text { Class) }\end{array}$ & Topology Description & $\begin{array}{l}\text { Number of } \\
\text { Branches }\end{array}$ & $\begin{array}{c}\text { Length of } \\
\text { Distribution Lines }\end{array}$ & $\begin{array}{c}\text { Length of } \\
\text { Branching Lines }\end{array}$ \\
\hline Urban case $\mathrm{A}$ & $\begin{array}{l}\text { Concatenated Typical OV } \\
\text { urban topology without hook }\end{array}$ & 3 & $\begin{aligned} L_{v, 1} & =500 \mathrm{~m}, \\
L_{v, 2} & =200 \mathrm{~m}, \\
L_{v, 3} & =100 \mathrm{~m}, \\
L_{v, 4} & =200 \mathrm{~m}\end{aligned}$ & $\begin{array}{c}L_{v, \mathrm{~b} 1}=8 \mathrm{~m} \\
L_{v, \mathrm{~b} 2}=13 \mathrm{~m} \\
L_{v, \mathrm{~b} 3}=10 \mathrm{~m}\end{array}$ \\
\hline Urban case B & $\begin{array}{l}\text { Concatenated aggravated OV } \\
\text { urban topology without hook }\end{array}$ & 5 & $\begin{array}{c}L_{v, 1}=200 \mathrm{~m}, \\
L_{v, 2}=50 \mathrm{~m}, \\
L_{v, 3}=100 \mathrm{~m}, \\
L_{v, 4}=200 \mathrm{~m}, \\
L_{v, 5}=300 \mathrm{~m}, \\
L_{v, 6}=150 \mathrm{~m}\end{array}$ & $\begin{array}{c}L_{v, \mathrm{~b} 1}=12 \mathrm{~m}, \\
L_{v, \mathrm{~b} 2}=5 \mathrm{~m}, \\
L_{v, \mathrm{~b} 3}=28 \mathrm{~m}, \\
L_{v, \mathrm{~b} 4}=41 \mathrm{~m}, \\
L_{v, \mathrm{~b} 5}=17 \mathrm{~m}\end{array}$ \\
\hline Suburban case & $\begin{array}{c}\text { Concatenated OV LV } \\
\text { suburban topology without } \\
\text { hook }\end{array}$ & 2 & $\begin{array}{l}L_{v, 1}=500 \mathrm{~m} \\
L_{v, 2}=400 \mathrm{~m} \\
L_{v, 3}=100 \mathrm{~m}\end{array}$ & $\begin{array}{l}L_{v, \mathrm{~b} 1}=50 \mathrm{~m} \\
L_{v, \mathrm{~b} 2}=10 \mathrm{~m}\end{array}$ \\
\hline Rural case & $\begin{array}{c}\text { Concatenated OV LV rural } \\
\text { topology without hook }\end{array}$ & 1 & $\begin{array}{l}L_{v, 1}=600 \mathrm{~m} \\
L_{v, 2}=400 \mathrm{~m}\end{array}$ & $L_{v, \mathrm{~b} 1}=300 \mathrm{~m}$ \\
\hline "LOS" case & $\begin{array}{l}\text { Concatenated OV LV Line- } \\
\text { of-Sight transmission } \\
\text { without hook }\end{array}$ & 0 & $L_{v, 1}=1000 \mathrm{~m}$ & - \\
\hline
\end{tabular}

when the number of concatenations $v$ may take integer values above 1 . Since the length of the indicative ad-hoc OV LV BPL topologies of Table 1 is equal to 1000 $\mathrm{m}$, the length of the modified ad-hoc OV LV BPL topologies of Table 3 becomes equal to $v \times 1000 \mathrm{~m}$.

\section{Numerical Results and Discussion}

The numerical results of this Section focus on evaluating the performance of HS-DET method after the installation of ad-hoc OV LV BPL topologies. Exploiting the already acquired knowledge of [20]-[22], the impact of the deviation from the initial measurement positions and of longer ad-hoc OV LV BPL topologies than the typical ones on the HS-DET method performance is assessed through the prism of $\triangle P E S$ and its appropriate contour plots. The goal of HS-DET method is to detect the hook style energy theft when the aforementioned two special issues of the operation of the 
ad-hoc OV LV BPL topologies occur. For that reason, a HS-DET method roadmap and $\triangle P E S$ thresholds of [20]-[22] are going to be exploited. As the circuital, topological and coupling scheme characteristics of OV LV BPL networks are concerned, these remain the same with [20]-[22].

\subsection{HS-DET Method and the Deviation from the Initial Measurement Positions}

With reference to eqs. (4)-(6), when the Information Technology department can exactly install its ad-hoc OV LV BPL topology at the initial measurement positions and no hook style energy theft occurs, $\triangle P E S$ values between the original ad-hoc OV LV BPL topology and the respective modified ad-hoc OV LV BPL topology without the hook are going to be equal to zero, which is anyway the obvious result. However, small measurement location deviations (e.g., from few $\mathrm{cm}$ to approximately 10 $\mathrm{m})$ from the initial measurement positions may occur having as a result a $\triangle P E S$ difference between the original ad-hoc OV LV BPL topology and the respective modified ad-hoc OV LV BPL topology without hook except for the "LOS" transmission case. In accordance with Sec. 3.2, there are two scenarios that should be examined.

\subsubsection{Scenario A}

With reference to Tables 1 and 2, let assume that a measurement location deviation devt occurs between the original ad-hoc OV LV BPL topologies and the respective shifted ad-hoc OV LV BPL topologies without hook that may range from $-10 \mathrm{~m}$ to $10 \mathrm{~m}$. Since Information Technology department can accurately measure the distance between the transmitting and receiving end and can assure that this distance remains equal to $D$, the measurement location deviation $d e v_{\mathrm{r}}$ is given by eq. (7). In Fig. 2, $\triangle P E S$ is plotted with respect to the measurement location deviation dev between the typical OV LV urban topology and the shifted typical OV LV urban topology without hook. In the same figure, same $\triangle P E S$ curves with the urban case A but for the urban case B, suburban case, rural case and "LOS" transmission case are given.

From Fig. 2, it is evident that extremely low $\triangle P E S$ values are observed regardless of the applied magnitude of measurement location deviations. Practically, a positive measurement location deviation can be treated as a MTL distribution cable segment that is cascaded to the examined original ad-hoc OV LV BPL topology. At the same time, the equal in absolute value but negative measurement location deviation, which is expressed in eq. (7), can be treated as a MTL distribution cable segment that is removed from the examined original ad-hoc OV LV BPL topology. In total, since the distance between the transmitting and receiving end of Scenario A always remains equal to $D$, the symmetry property of BPL channels, which has been detailed in [50], [51], holds regardless of the examined ad-hoc OV LV BPL topology. Therefore, small measurement location deviations in Scenario A cannot trigger a false hook style energy theft alarm since $\triangle P E S$ values remain significantly lower than the strict $10 \% \triangle P E S$ threshold.

Actually, it is expected that the small measurement location deviations of Scenario A cannot abrupt the high performance of HS-DET method in detecting the hook style energy theft. In order to validate the detection efficiency of HS-DET method when a hook style energy theft occurs, $\triangle(\triangle P E S)$ is going to be computed and presented hereafter; $\triangle(\triangle P E S)$ expresses the difference between the $\triangle P E S$ when a measurement location deviation occurs and the $\triangle P E S$ without the measurement location deviation -see Figs. 3(a)-(e) of [20]- for given ad-hoc OV LV BPL topology, hook distance from 
the transmitting end span and hook length span. In Fig. 3(a), $\triangle(\triangle P E S)$ is plotted versus the hook distance from the transmitting end and the hook length when the indicative ad-hoc OV LV BPL topology of urban case A is assumed, measurement location deviation $d e v_{\mathrm{t}}$ is equal to $-10 \mathrm{~m}$ and Scenario A is examined. In Figs. 3(b), 3(c), 3(d) and 3(e), same plots with Fig. 3(a) are given but for the case of the urban case B, suburban case, rural case and "LOS" case, respectively. In Figs. 4(a)-(e), same plots with the respective Figs. 3(a)-(e) but for the case of the measurement location deviation $d e v_{t}$ that is equal to $10 \mathrm{~m}$. Here, it should be noted that the hook distance span and

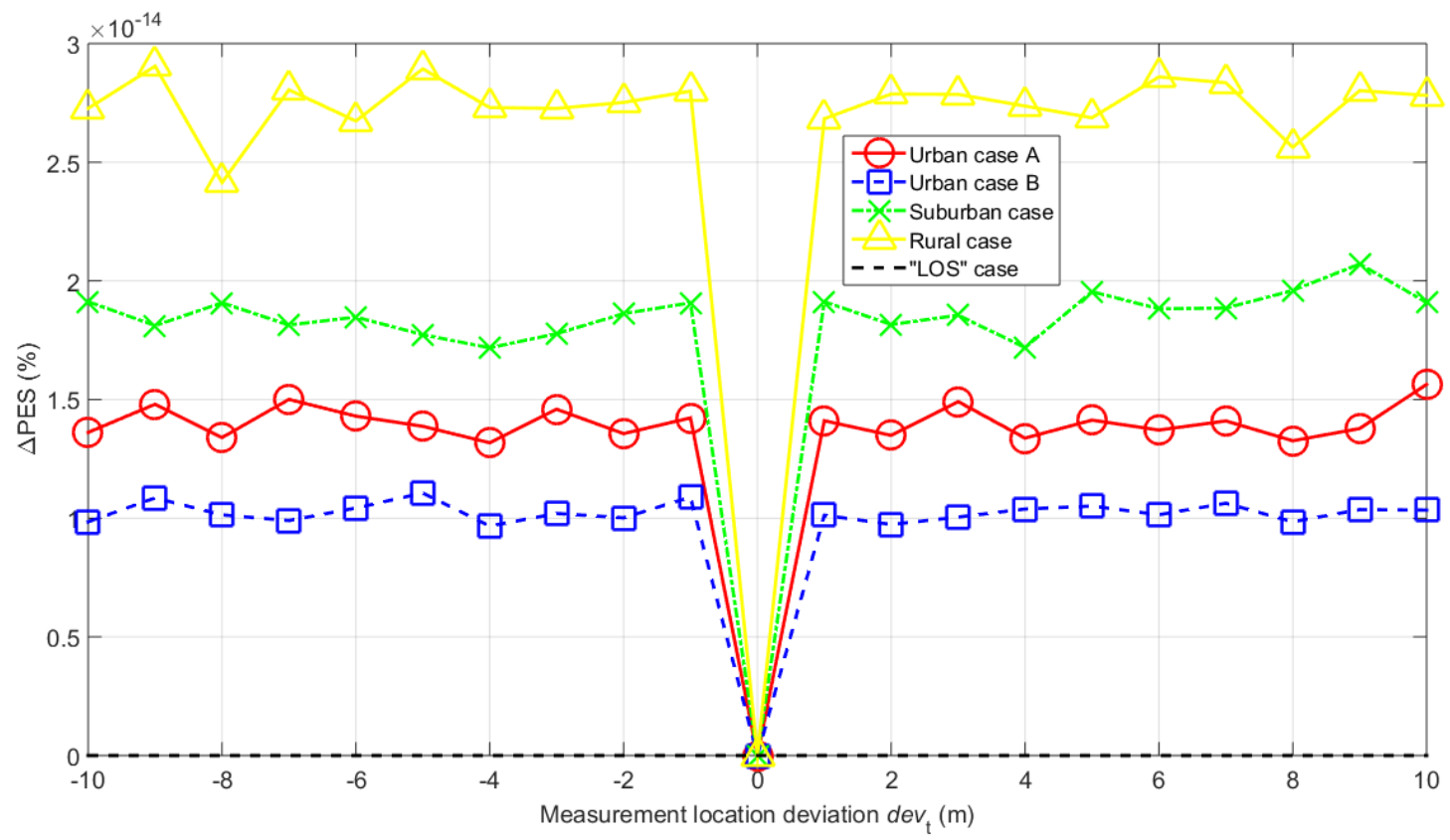

Fig. 2. $\triangle P E S$ of HS-DET method for the five indicative ad-hoc OV LV BPL topologies for various measurement location deviations $d e v_{\mathrm{t}}$ when no hook style energy theft occurs (Scenario A). 


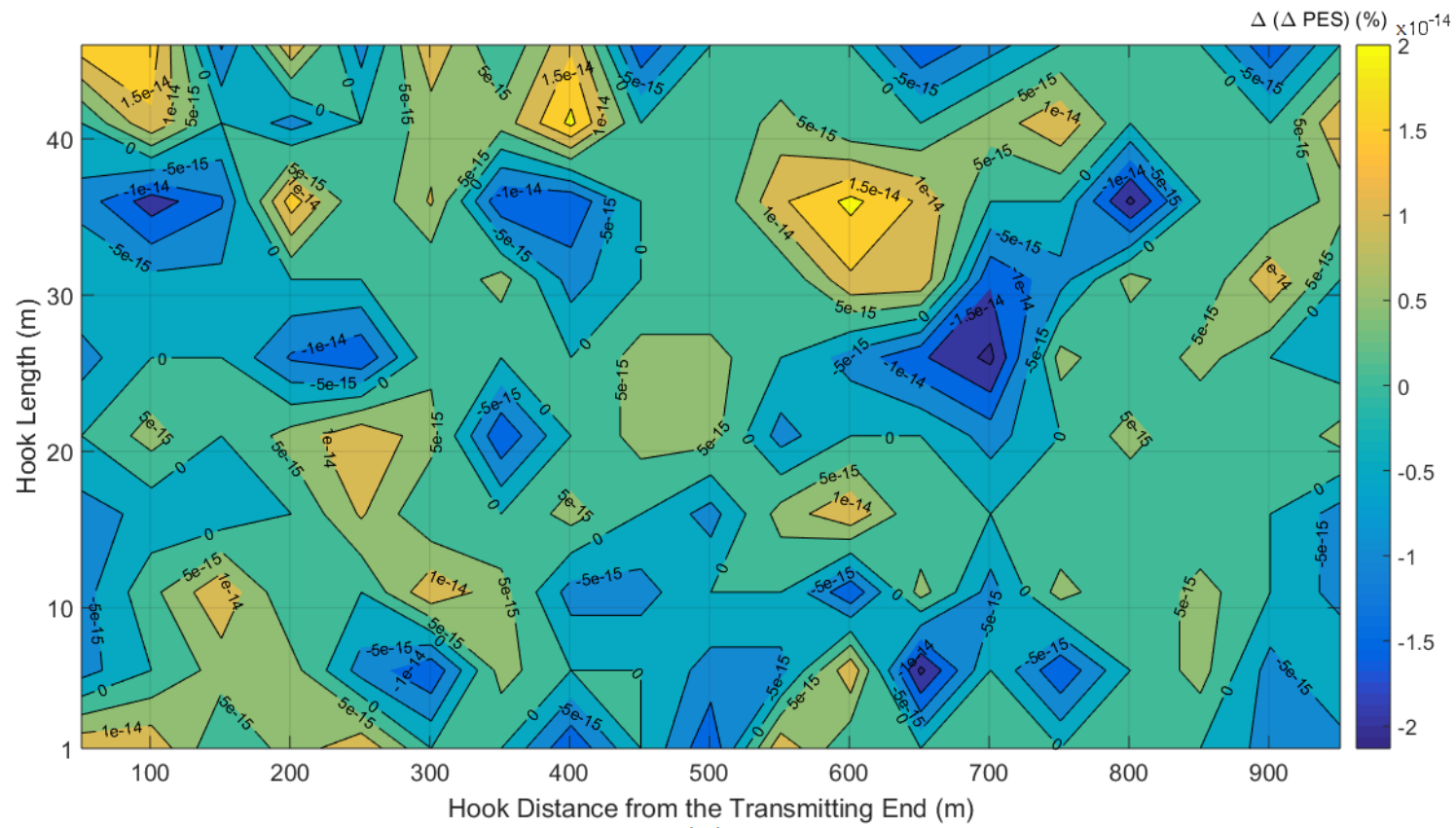

(a)

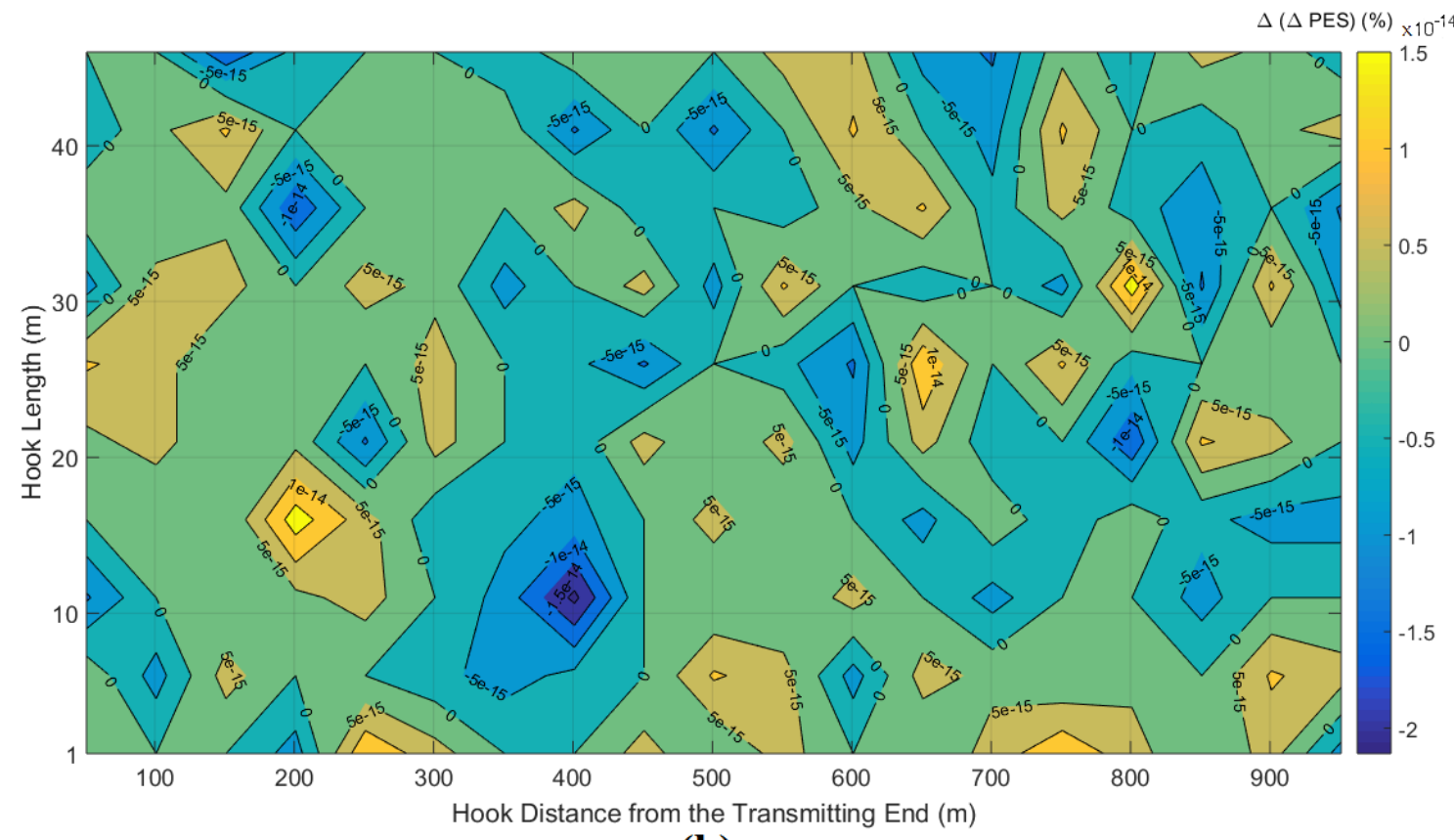

(b) 


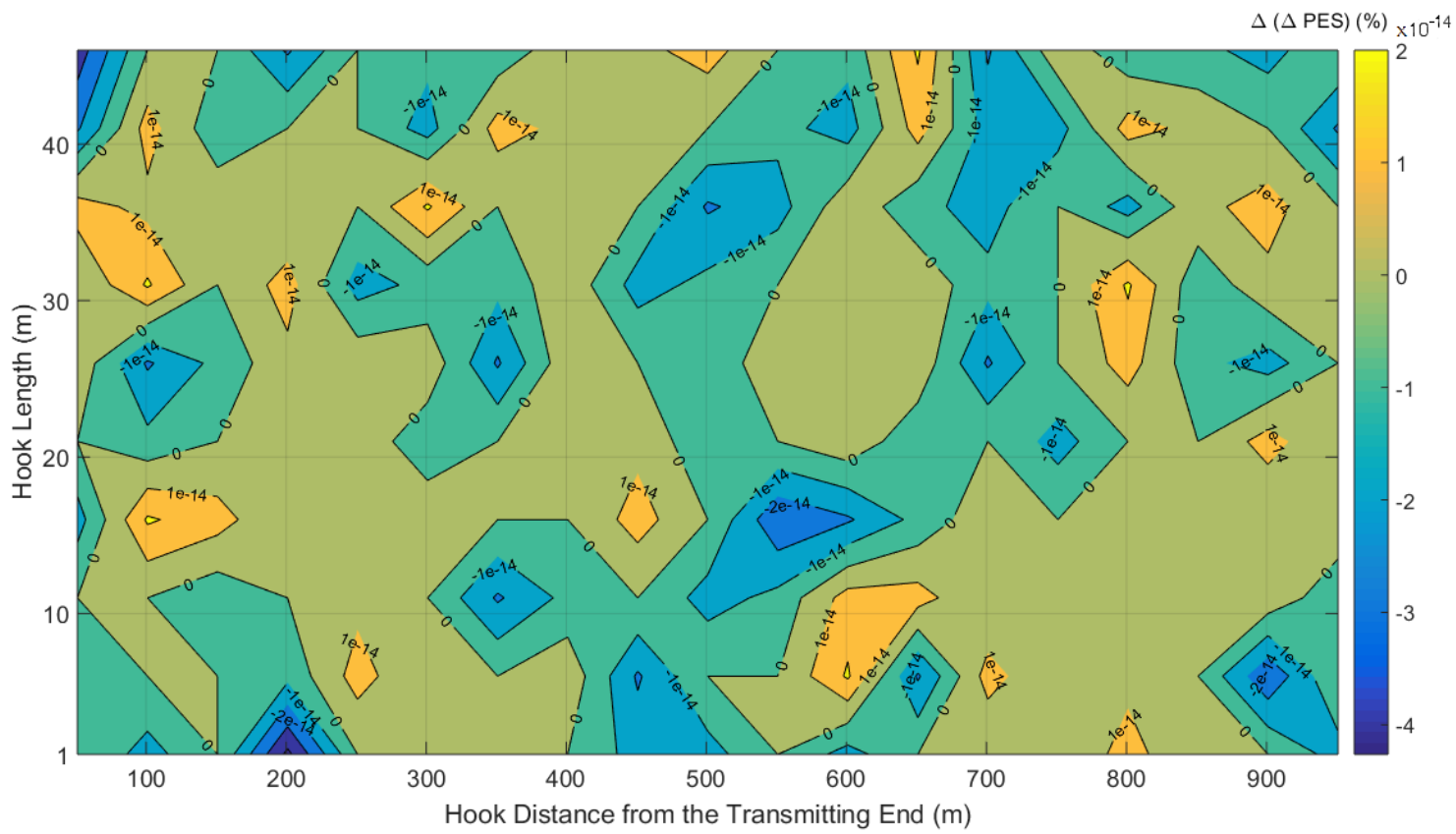

(c)

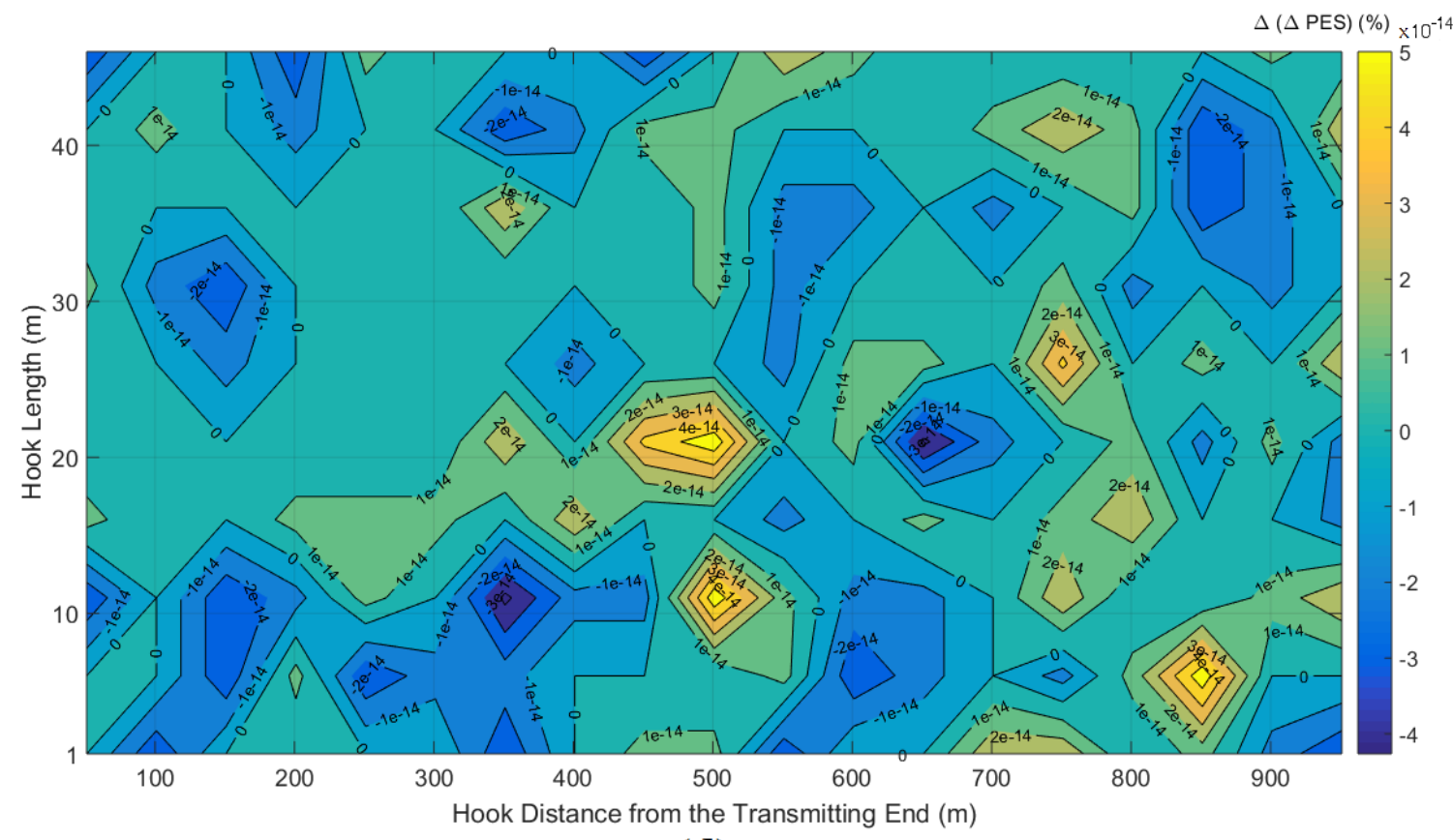

(d) 


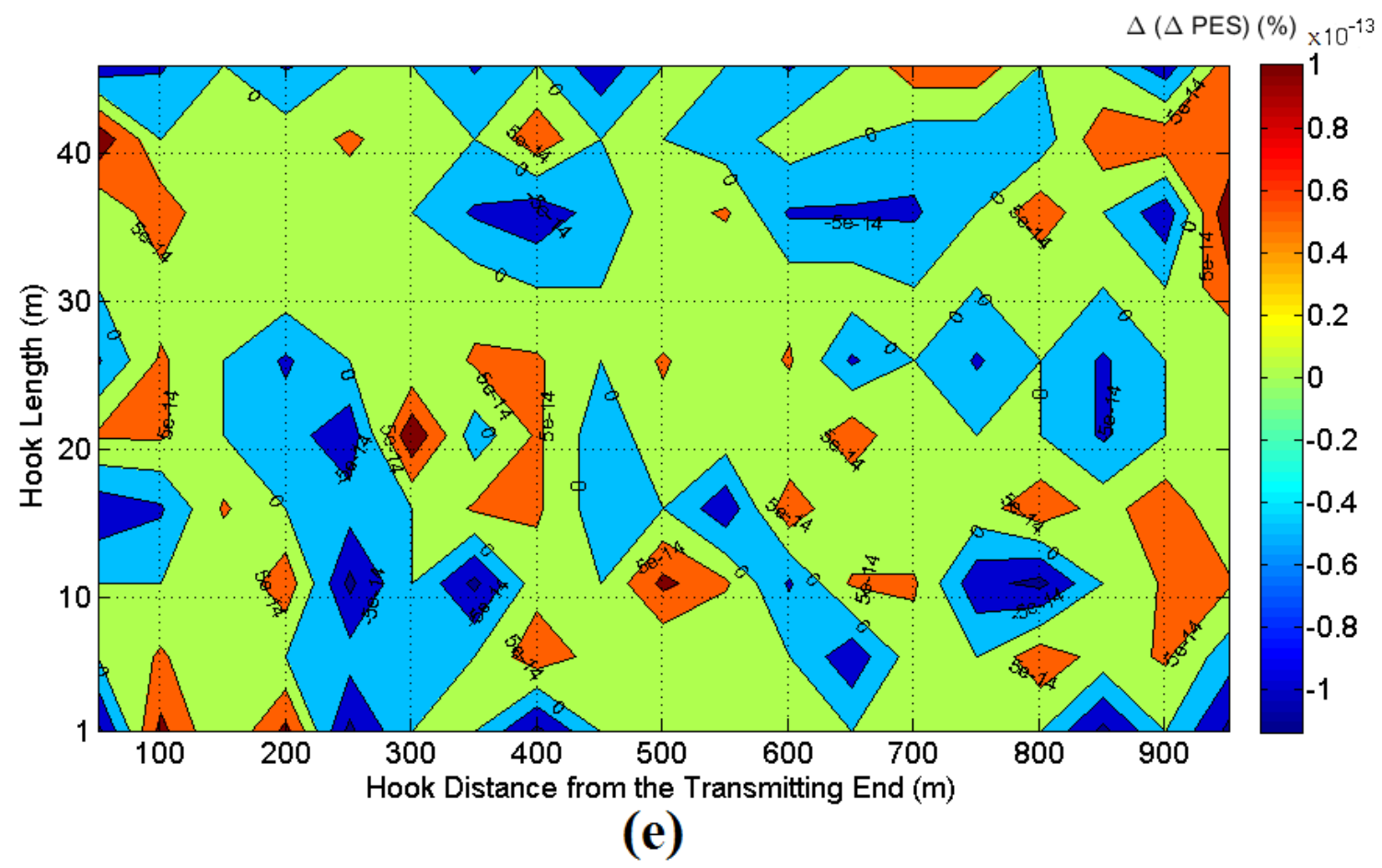

Fig. 3. $\triangle(\triangle P E S)$ of the indicative OV LV BPL topologies for various hook distances from the original transmitting end and hook lengths when measurement location deviation $d e v_{t}$ is equal to $-10 \mathrm{~m}$ (Scenario A). (a) Urban case A. (b) Urban case B. (c) Suburban case. (d) Rural case. (e) "LOS" case. 


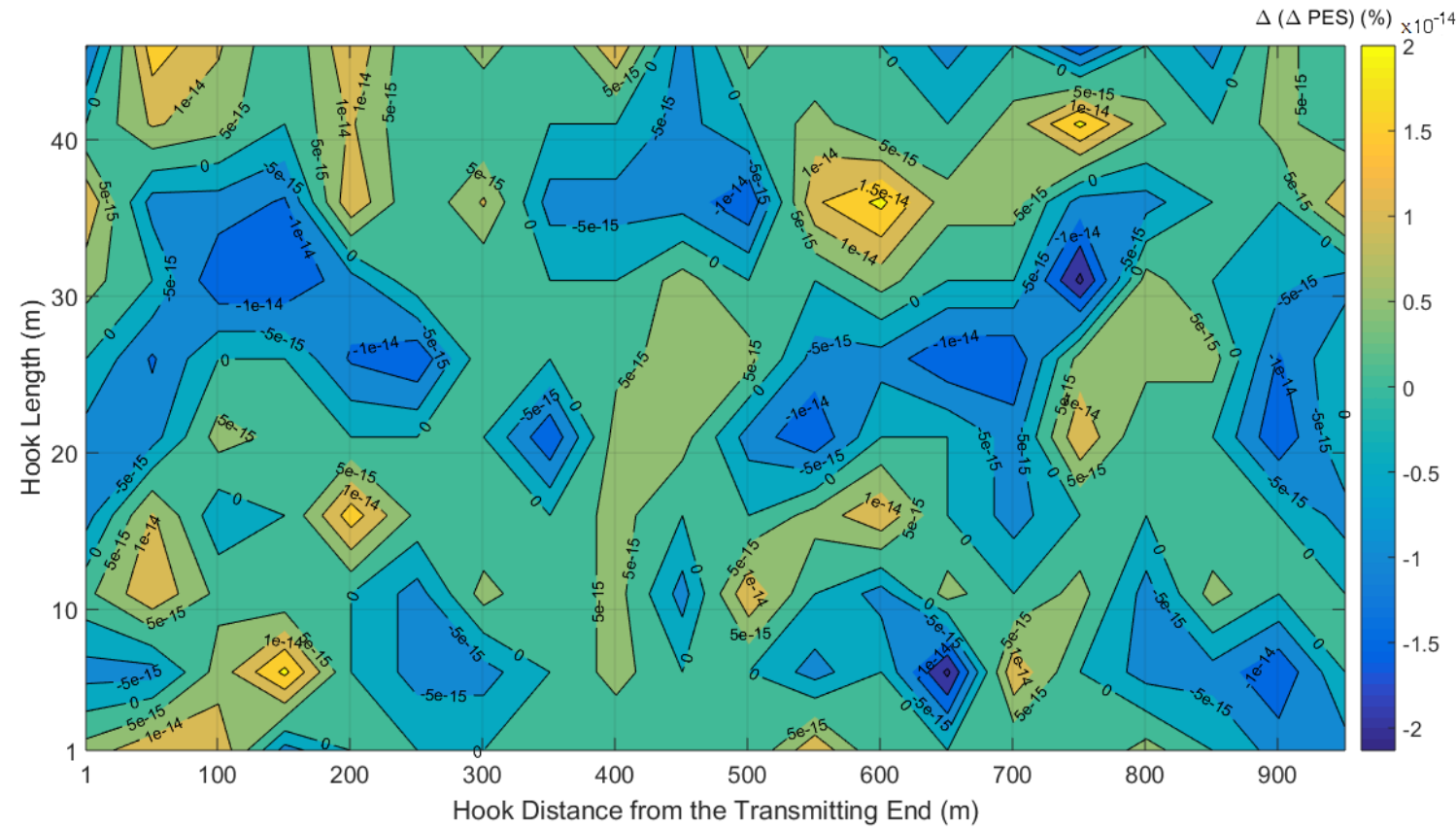

(a)

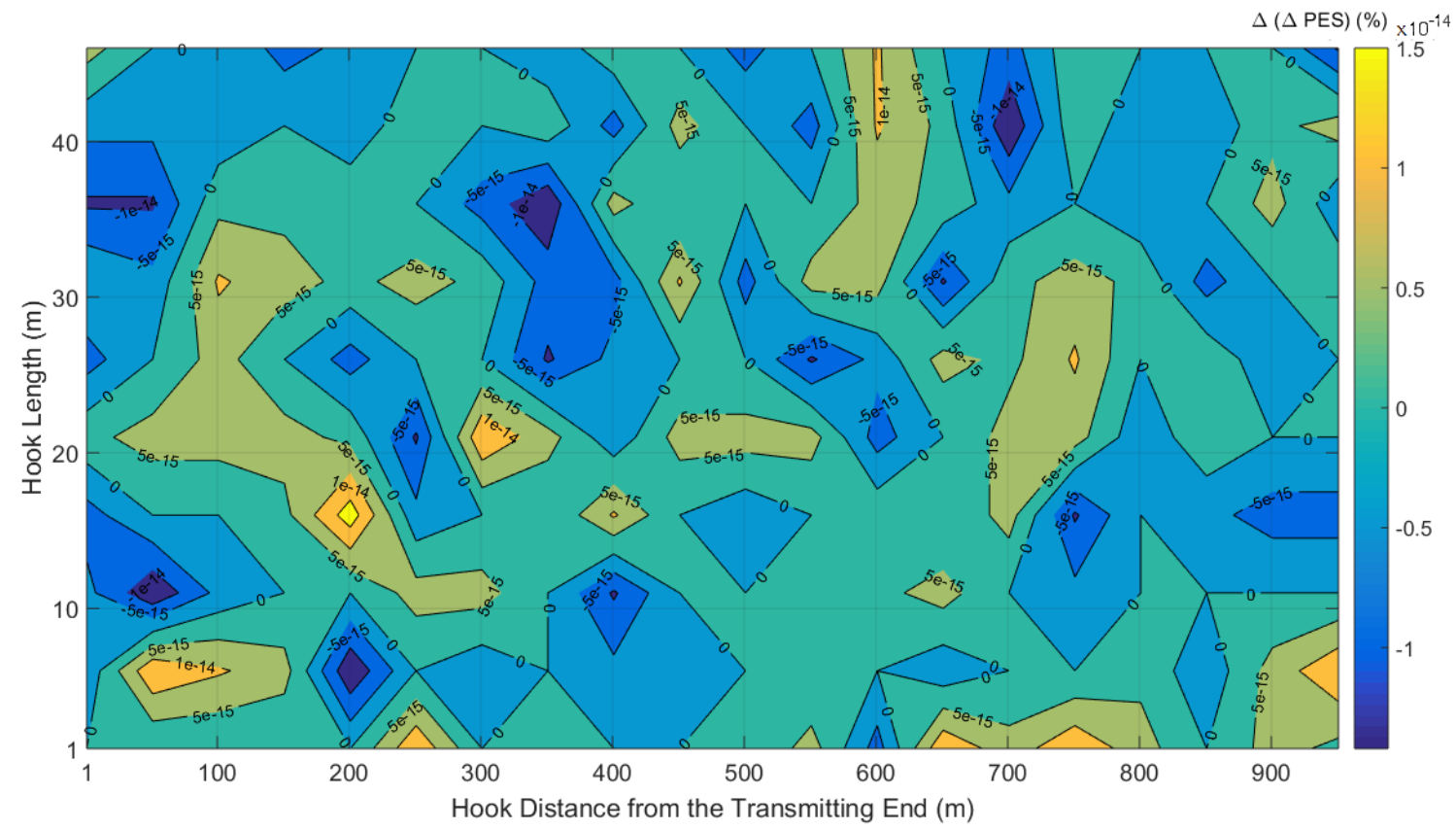

(b) 


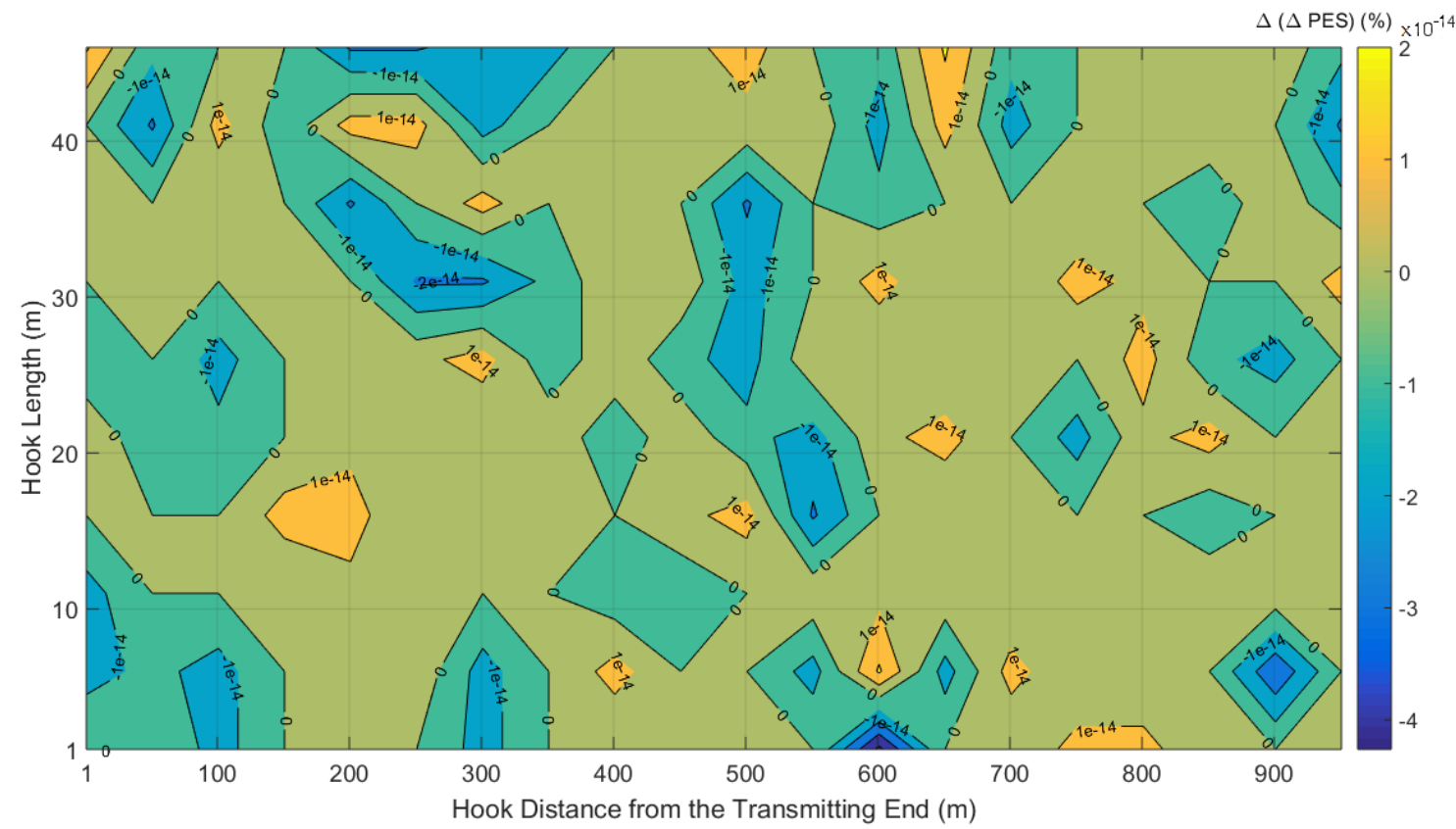

(c)

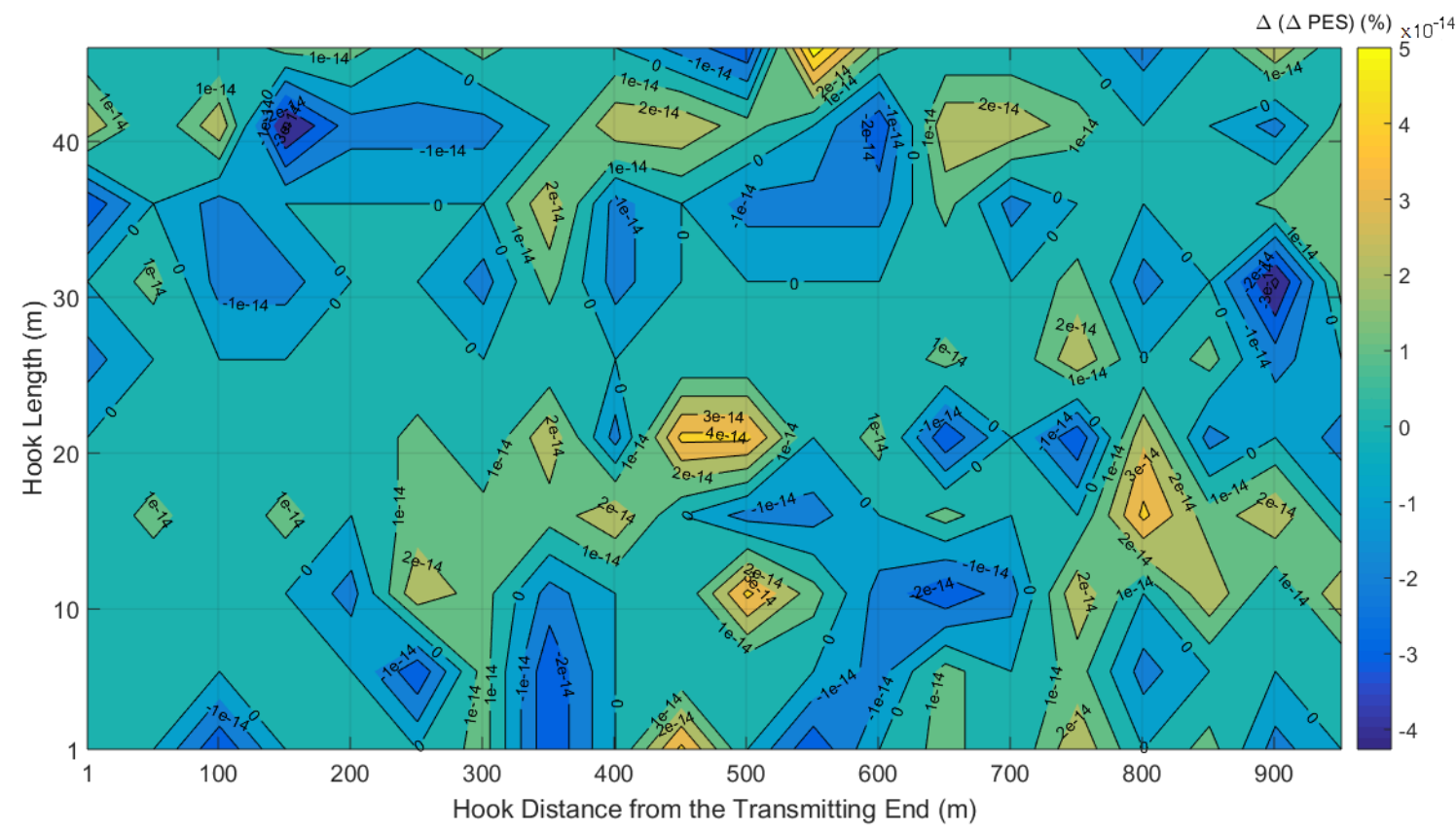

(d) 


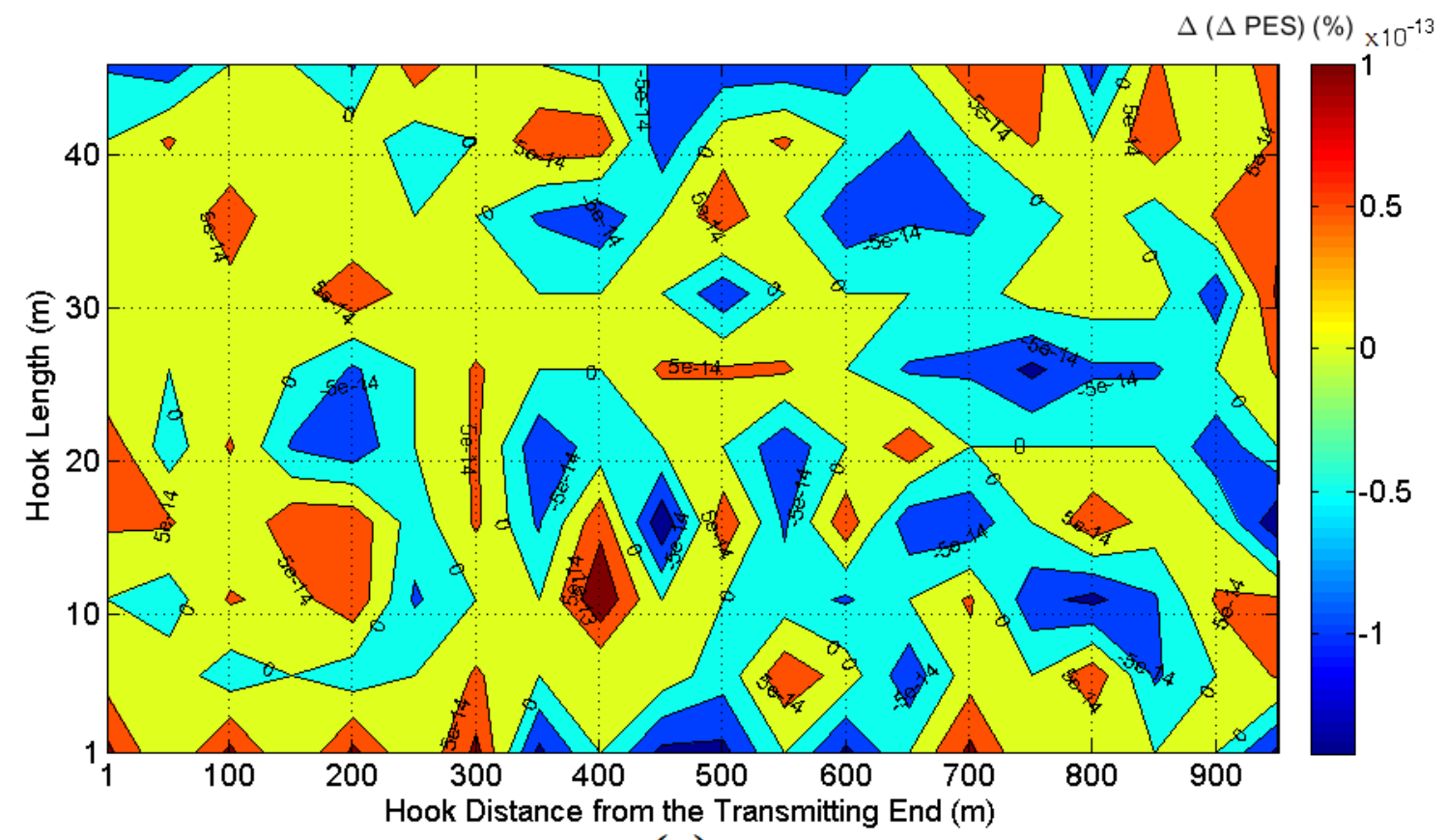

(e)

Fig. 4. Same curves with Fig. 3 but for measurement location deviation $d e v_{\mathrm{t}}$ of $10 \mathrm{~m}$.

the hook length span are assumed to be equal to $50 \mathrm{~m}$ and $5 \mathrm{~m}$, respectively, while the range of the hook distance from the transmitting end of the examined original ad-hoc OV LV BPL topology and the range of the hook length are from $1 \mathrm{~m}$ to $951 \mathrm{~m}$ and from $1 \mathrm{~m}$ to $46 \mathrm{~m}$, respectively, for all the examined contour plots of this paper. The selection of the previous plot specifications has been done for the sake of the direct PES submetric comparison against Figs. 3(a)-(e) of [20].

By comparing Figs. 3(a)-(e) and 4(a)-(e) with Figs. 3(a)-(e) of [20], several interesting comparative observations can be made regarding the performance of HS-DET method when Scenario A is adopted, namely:

- Regardless of the measurement location deviation $d e v_{\mathrm{t}}$ and its dependent measurement location deviation $d e v_{\mathrm{r}}, \triangle(\triangle P E S)$ remains extremely low in all the examined cases of Scenario A, in the order of $10^{-14} \%$. This indicates that the small values of $\triangle P E S$, which have already been spotted in Fig. 2, are further reflected on respective small $\triangle(\triangle P E S)$ values of Figs. 3(a)-(e) and 4(a)-(e) thus unveiling the invulnerability of HS-DET method when various measurement location deviations that are in agreement with Scenario A occur.

- Since $\triangle P E S$ of Figs. 3(a)-(e) of [20] remains well above the $10 \%$ threshold of the easy hook style energy theft detection and all the observed $\triangle(\triangle P E S)$ values of Figs. 3(a)-(e) and 4(a)-(e) remain in the order of $10^{-14} \%, \triangle P E S$ values of the modified ad-hoc OV LV BPL topologies after the hook insertion will remain significantly greater than the strict $\triangle P E S$ threshold of $10 \%$ thus allowing the easy hook style energy theft detection regardless of the magnitude of the measurement location deviations.

- From Figs. 3(a)-(e) and 4(a)-(e), $\triangle(\triangle P E S)$ values remain in the order of $10^{-14} \%$ regardless of the examined ad-hoc OV LV BPL topology. Similarly to [20], 
the hook detection becomes easier in the cases of ad-hoc OV LV BPL topologies of low number of long branches such as "LOS" and rural topologies. In the latter cases, a hook insertion provokes the birth of a multipath environment which is accompanied by deep and frequent spectral notches to the measured coupling scheme channel attenuation and, of course, significant increase of $P E S_{\text {mod }}$ and $\triangle P E S$. Indeed, the easy hook detection is validated by the high $\triangle \mathrm{PES}$ values across the contour plots of the "LOS" and rural OV LV BPL topologies of [20].

- From Figs. 3(a)-(e) and 4(a)-(e), $\triangle(\triangle P E S)$ values remain in the order of $10^{-14} \%$ regardless of the hook length. Similarly to [20], the detection of the hook style energy theft of Scenario A in ad-hoc OV LV BPL topologies remains easier when the hook remains short enough (i.e., shorter than $10 \mathrm{~m}$ ) while the detection becomes less easy but safe when the hook exceeds $10 \mathrm{~m}$.

- From Figs. 3(a)-(e) and 4(a)-(e), $\triangle(\triangle P E S)$ values remain in the order of $10^{-14} \%$ regardless of the hook position during the Scenario A. Similarly to [20], the hook distance from the transmitting end becomes more crucial in ad-hoc OV LV BPL topologies with high number of branches rather than in the ad-hoc OV LV BPL rural and "LOS" topologies.

From the previous observations, it is highlighted the main advantage of Scenario A that is the fixed distance between the transmitting and receiving end. This implies that even if large measurement location deviations dev $v_{\mathrm{t}}$ may occur, these deviations can be counterbalanced by the equal in magnitude but opposite measurement location deviations dev r. Hence, the most important issue during the installation of an ad-hoc OV LV BPL topology for the hook style energy theft detection by the Information Technology department is the maintenance of the same distance between the transmitting and receiving end rather than the exact installation position of the transmitting end since measurement location deviations $d e v_{\mathrm{t}}$ and $d e v_{\mathrm{r}}$ can be counterbalanced.

\subsubsection{Scenario $B$}

With reference to Tables 1 and 2, let assume that a measurement location deviation $d e v_{\mathrm{t}}$ occurs between the original ad-hoc OV LV BPL topologies and the respective shifted ad-hoc OV LV BPL topologies without hook that may range from $-10 \mathrm{~m}$ to $10 \mathrm{~m}$. In Scenario B, it is assumed that Information Technology department cannot assure that the distance between the transmitting and receiving end is equal to $D$. Since the length of the shifted ad-hoc OV LV BPL topology is not exactly known, the measurement location deviation $d e v_{\mathrm{r}}$ may also range from $-10 \mathrm{~m}$ to $10 \mathrm{~m}$ independently of the measurement location deviation $d e v \mathrm{t}$.

With reference to Sec. 4.1.1, it has been verified that the main issue regarding the performance of HS-DET method is the algebraic sum of the measurement location deviations $d e v_{\mathrm{t}}$ and $d e v_{\mathrm{r}}$ rather than their exact values. Hence, the measurement location deviation $d e v_{\mathrm{t}}$ can be considered to vary from $-20 \mathrm{~m}$ to $20 \mathrm{~m}$ in the following analysis so that all the different combinations of the measurement location deviations $d e v_{\mathrm{t}}$ and $d e v_{\mathrm{r}}$ can equivalently be studied without creating additional complexity. In Fig. 5, $\triangle P E S$ is plotted with respect to the measurement location deviation dev between the typical OV LV urban topology and the shifted typical OV LV urban topology without hook when Scenario B is applied. In the same figure, same $\triangle P E S$ curves with the urban 
case A but for the urban case B, suburban case, rural case and "LOS" transmission case are given.

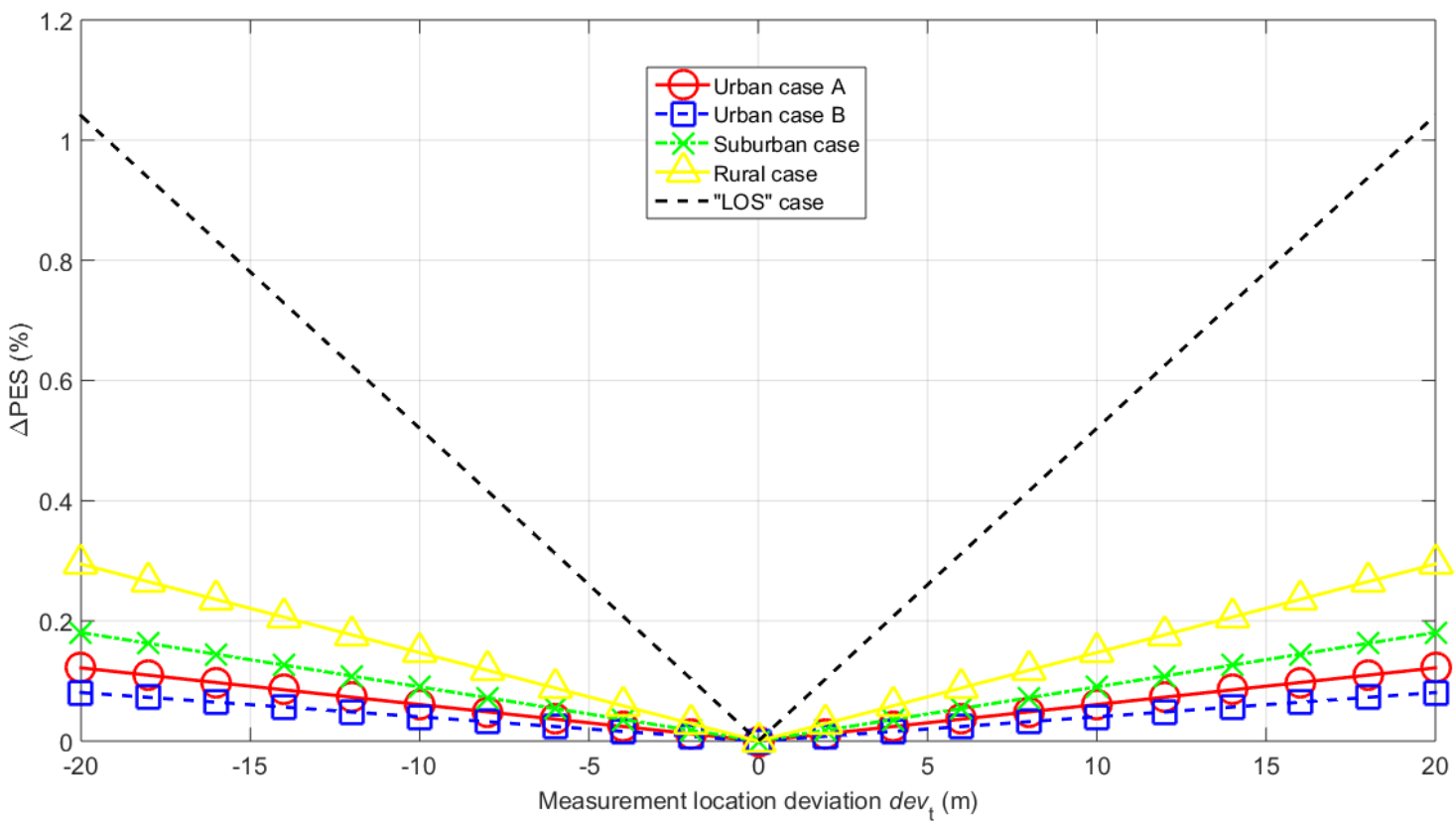

Fig. 5. $\triangle P E S$ of HS-DET method for the five indicative ad-hoc OV LV BPL topologies for various measurement location deviations $d e v_{\mathrm{t}}$ when no hook style energy theft occurs (Scenario B).

From Fig. 5, low $\triangle P E S$ values that depend on the applied magnitude of measurement location deviation $d e v_{\mathrm{t}}$ are observed in Scenario B. By comparing Figs. 2 and 5, $\triangle P E S$ values of Scenario $B$ are significantly higher than the ones of Scenario A but anyway remain significantly lower than the strict $10 \% \triangle P E S$ threshold. This is a logical result since insertion or removal of MTL cable segments are considered for the ad-hoc OV LV BPL topologies when positive or negative measurement location deviations $d e v_{\mathrm{t}}$ are examined. Note that the symmetry property of BPL channels, which holds in Scenario A, does not stand in Scenario B except for the case where measurement location deviation $d e v_{\mathrm{t}}$ is equal to 0 . In the latter case, the study of Scenario B degenerates into the study of Scenario A.

In accordance with [23], [25], [27], [30], [48], the small $\triangle P E S$ values of Fig. 5 are explained by the fact that multipath rather than "LOS" transmission distance is identified as the dominant factor in OV LV BPL channels thus mainly affecting BPL signal attenuation. Hence, the effect of insertion or removal of MTL cable segments at the ad-hoc OV LV BPL topologies is not so severe and is not recognized as the primary attenuation factor since multipath attenuation is the primary one. Therefore, among $\triangle P E S$ values of different ad-hoc OV LV BPL topologies in Fig. 5, the most affected ones by the measurement location deviations of Scenario B are the "LOS" and rural OV LV BPL topologies since multipath attenuation remains negligible in these cases.

In addition, it should be noted that $\triangle P E S$ values of ad-hoc OV LV BPL topologies remain negligible with respect to the strict $10 \% \triangle P E S$ threshold while they start to become important with respect to the loose $0 \%$ $\triangle P E S$ threshold of [21], 
In fact, if the loose $0 \% \triangle P E S$ threshold, but risky in terms of a possible false alarm, is assumed only the "LOS" ad-hoc OV LV BPL topology can trigger this false alarm (i.e., $\triangle P E S$ values slightly greater than $1 \%$ when the absolute value of measurement location deviation $d e v_{\mathrm{t}}$ is equal to $20 \mathrm{~m}$ ) since the other ad-hoc OV LV BPL topologies are characterized by significantly low $\triangle P E S$ values (i.e., $\triangle P E S$ values lower than $0.3 \%$ ).

Similarly to Scenario A, it is expected that the small measurement location deviations of Scenario B cannot foment the high performance of HS-DET method against the hook style energy theft. In order to validate the detection efficiency of HS-DET method when a hook style energy theft occurs, $\triangle(\triangle P E S)$ is going to be computed and presented for Scenario B; in Fig. 6(a), $\triangle(\triangle P E S)$ is plotted versus the hook distance from the transmitting end and the hook length when the indicative ad-hoc OV LV BPL topology of urban case A is assumed, measurement location deviation $d e v_{\mathrm{t}}$ is equal to $-20 \mathrm{~m}$ and Scenario B is applied. In Figs. 6(b), 6(c), 6(d) and 6(e), same plots with Fig. 6(a) are given but for the case of the urban case B, suburban case, rural case and "LOS" case, respectively. In Figs. 7(a)-(e), same plots with the respective Figs. 6(a)-(e) but for the case of the measurement location deviation $d e v_{t}$ that is equal to $20 \mathrm{~m}$ are given. As the hook characteristics are concerned for the examined contour plots, these are the same with Figs. 3(a)-(e) and 4(a)-(e).

By comparing Figs. 6(a)-(e) and 7(a)-(e) with Figs. 3(a)-(e), 4(a)-(e) and 3(a)-(e) of [20], additional observations can be made regarding the performance of HSDET method when Scenario B is examined, namely:

- Similarly to Figs. 3(a)-(e), the first value of the hook distance from the original transmitting end in Figs. 6(a)-(e) is equal to $51 \mathrm{~m}$. Since a measurement location deviation $d e v_{\mathrm{t}}$ of $-10 \mathrm{~m}$ and $-20 \mathrm{~m}$ is assumed in Figs. 3(a)-(e) and Figs. 6(a)-(e), respectively, this is equal to a respective MTL cable segment removal of $10 \mathrm{~m}$ and $20 \mathrm{~m}$ from the transmitting end of the original ad-hoc OV LV BPL topologies with reference to Table 2. Since the range of the hook distance from the transmitting end of the examined original ad-hoc OV LV BPL topology is equal to $1 \mathrm{~m}$, the hook distance from the original transmitting end span is equal to $50 \mathrm{~m}$ and the first value of the range of the hook distance from the transmitting end cannot be used, the first value of the hook distance from the original transmitting end in Figs. 3(a)-(e) and 6(a)-(e) is equal to 51m.

- Negative $\triangle(\triangle P E S)$ values are observed in Figs. 6(a)-(e) whereas positive $\triangle(\triangle P E S)$ values are observed in Figs. 7(a)-(e). Conversely to Figs. 3(a)-(e) and 4(a)-(e) where a mixed scenario regarding the sign of $\triangle(\triangle P E S)$ occurs, the negative $\triangle(\triangle P E S)$ values but of small magnitudes that are observed in Figs. 6(a)-(e) indicate that HS-DET method slightly more difficult detects the hook style energy theft; the opposite occurs when the positive $\triangle(\triangle P E S)$ values are demonstrated in Figs. 7(a)-(e). Hence, positive measurement location deviations that entail larger length between the transmitting and receiving end of the modified ad-hoc OV LV BPL topologies can facilitate the detection of hook style energy theft.

- In accordance with [20], the hook distance from the transmitting end influences the performance of HS-DET method only in the cases of ad-hoc OV LV BPL topologies with high number of branches thus creating areas of different hook style energy theft detection difficulty in contour plots. Hence, with reference to Figs. 3(a)-(e) of [20], higher $\triangle \mathrm{PES}$ values characterize areas of easier hook style 
energy theft detection. With reference to Figs. 6(a)-(e) and 7(a)-(e),

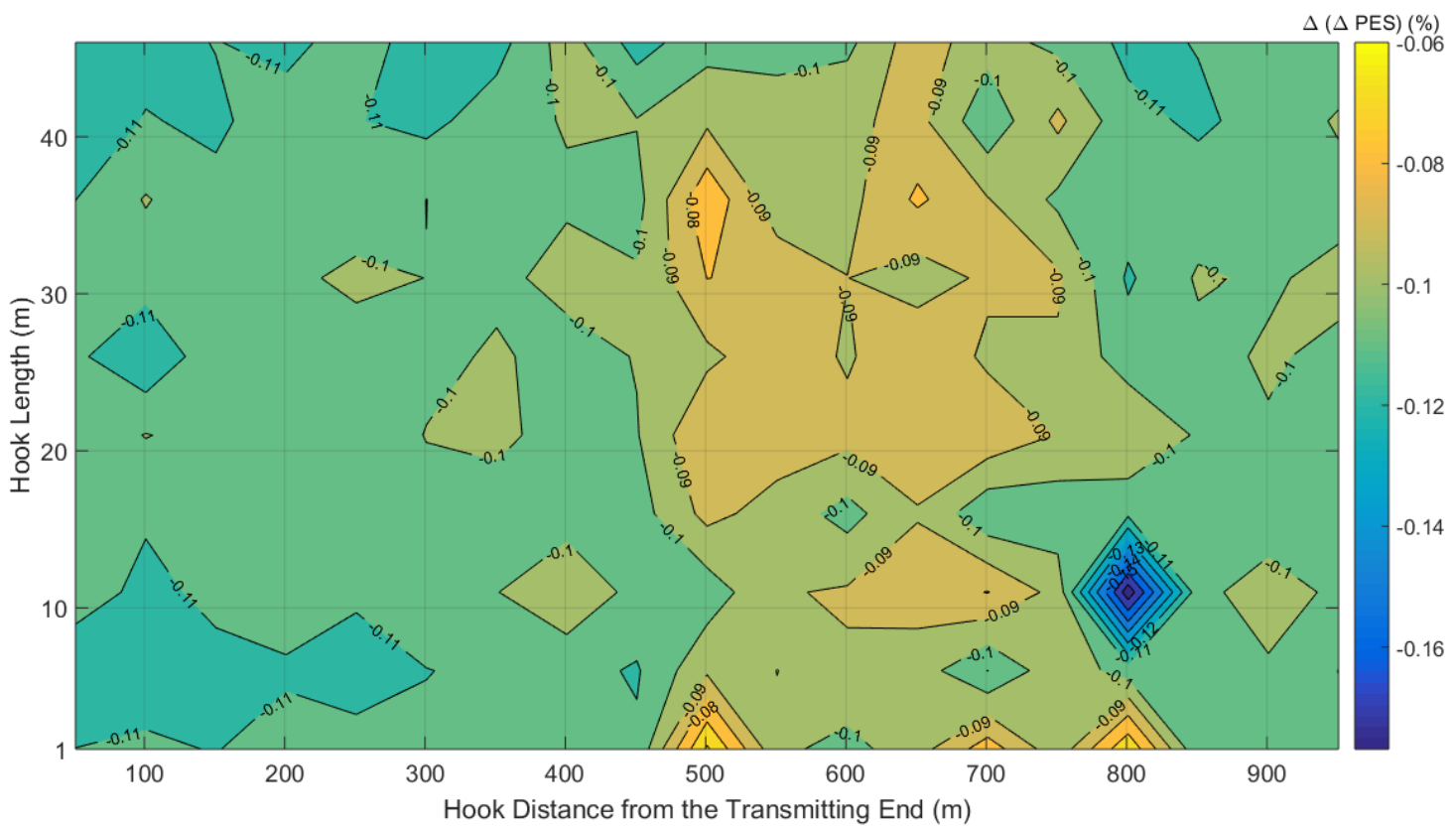

(a)

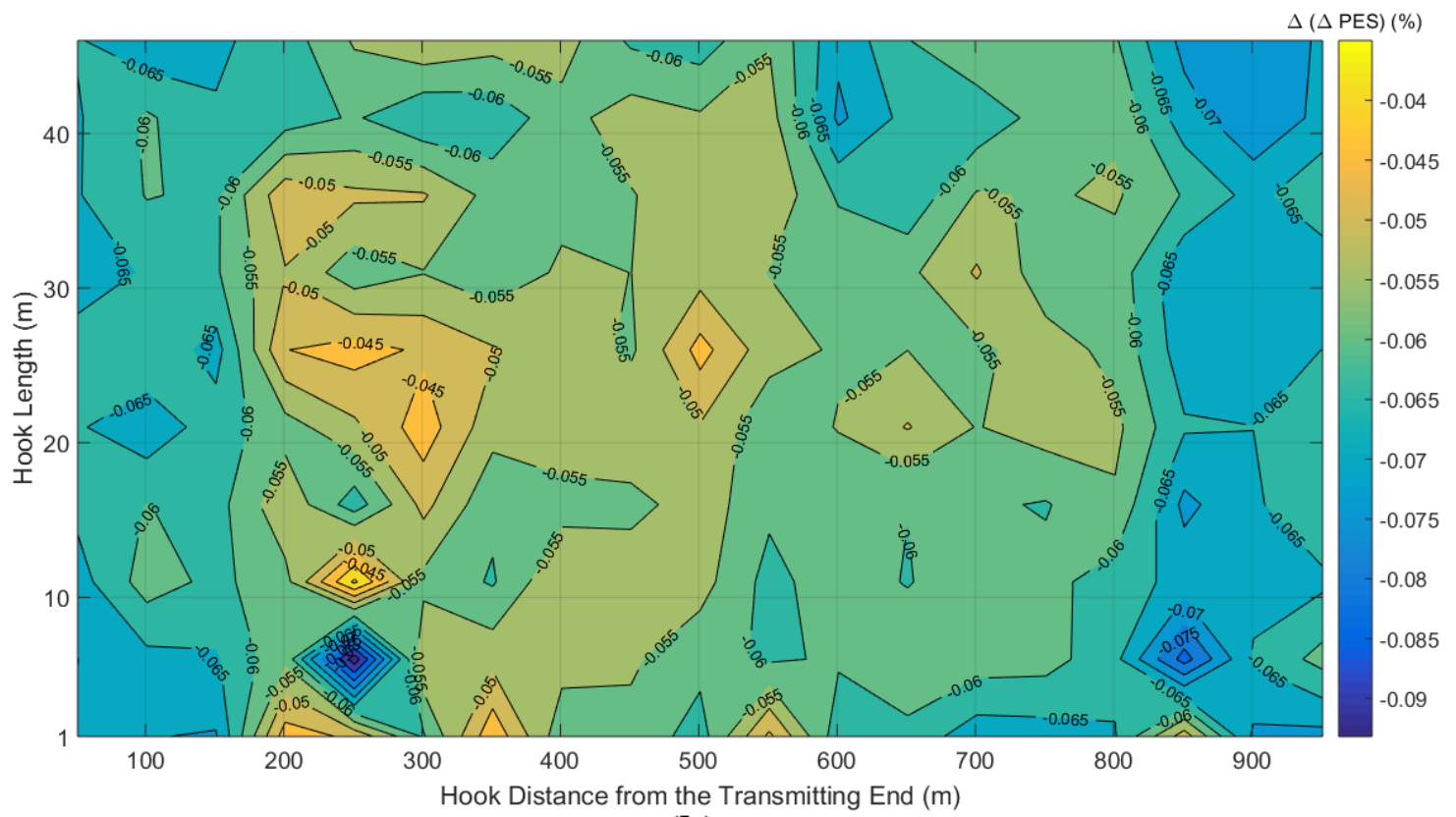

(b) 


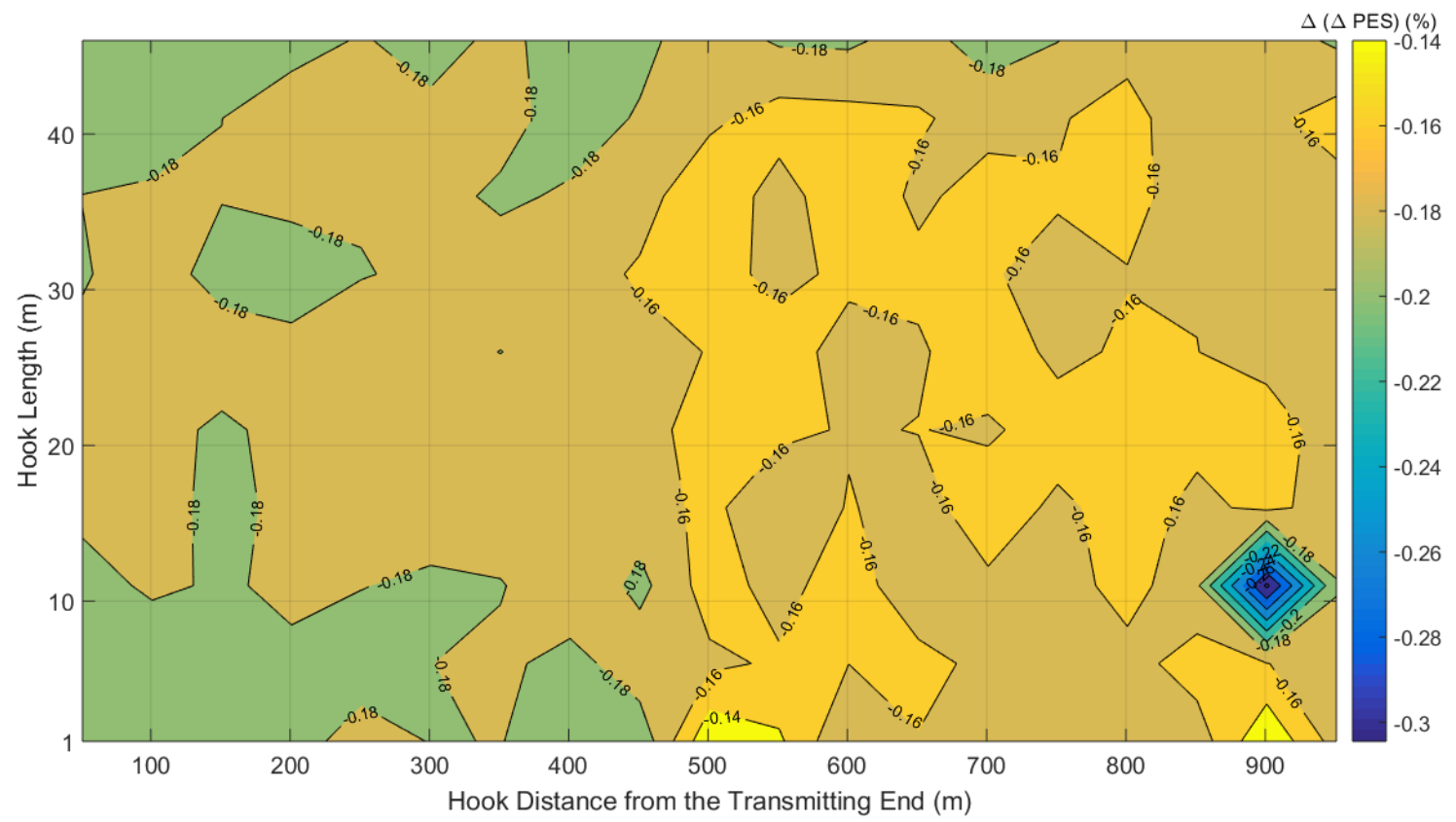

(c)

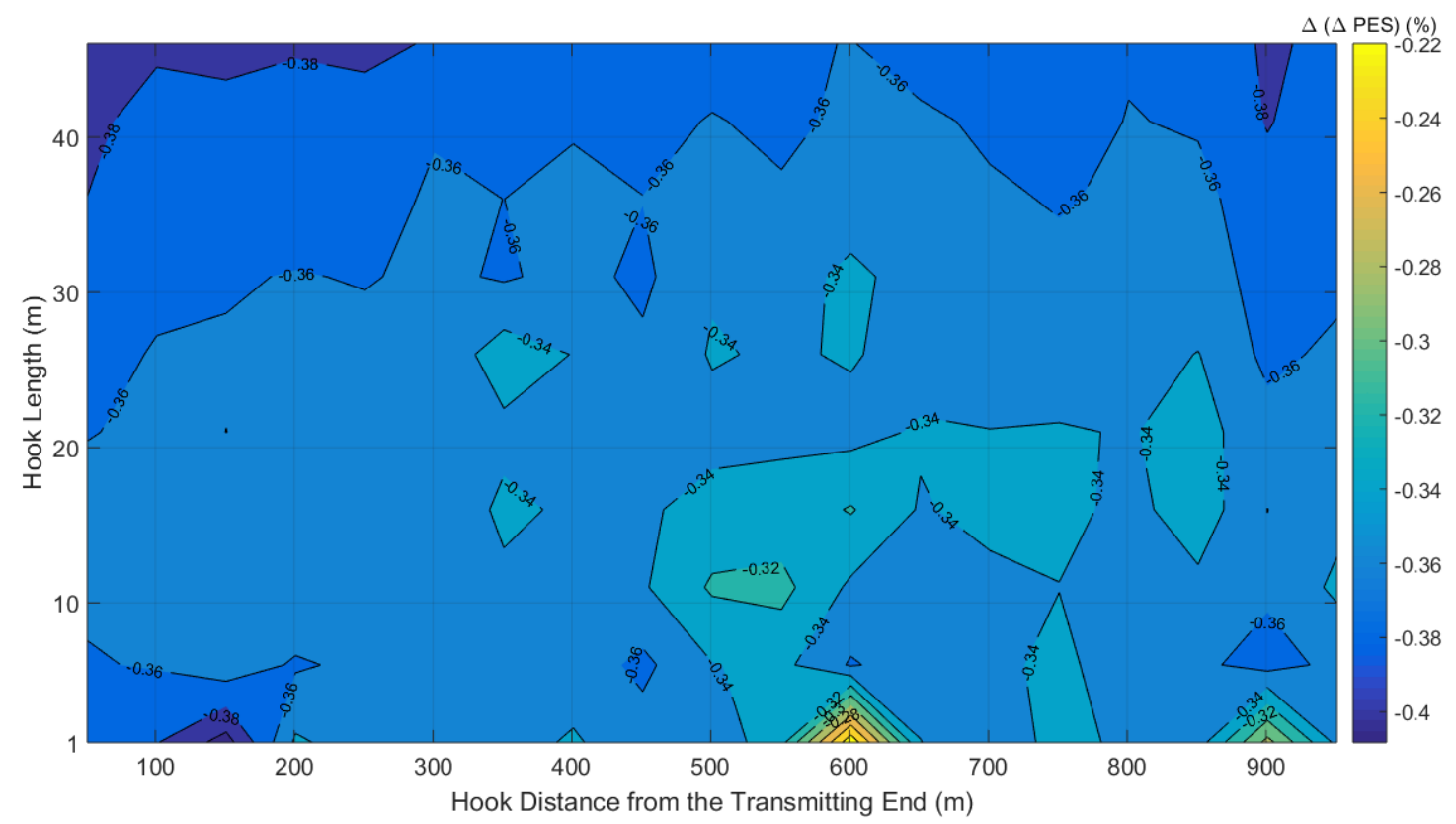

(d) 


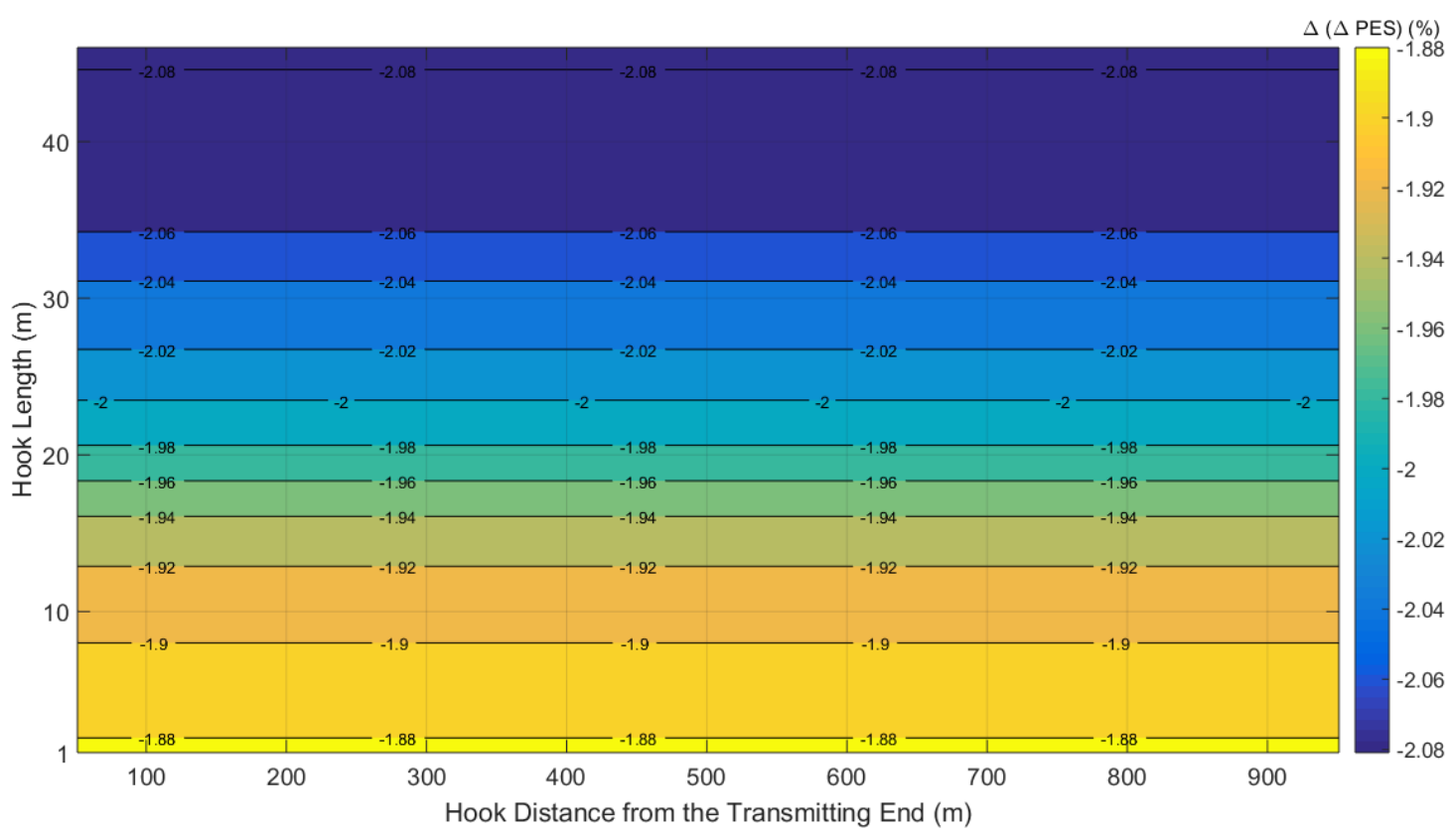

(e)

Fig. 6. $\triangle(\triangle P E S)$ of the indicative OV LV BPL topologies for various hook distances from the original transmitting end and hook lengths when measurement location deviation $d e v_{\mathrm{t}}$ is equal to $-20 \mathrm{~m}$ (Scenario B). (a) Urban case A. (b) Urban case B. (c) Suburban case. (d) Rural case. (e) "LOS" case. 


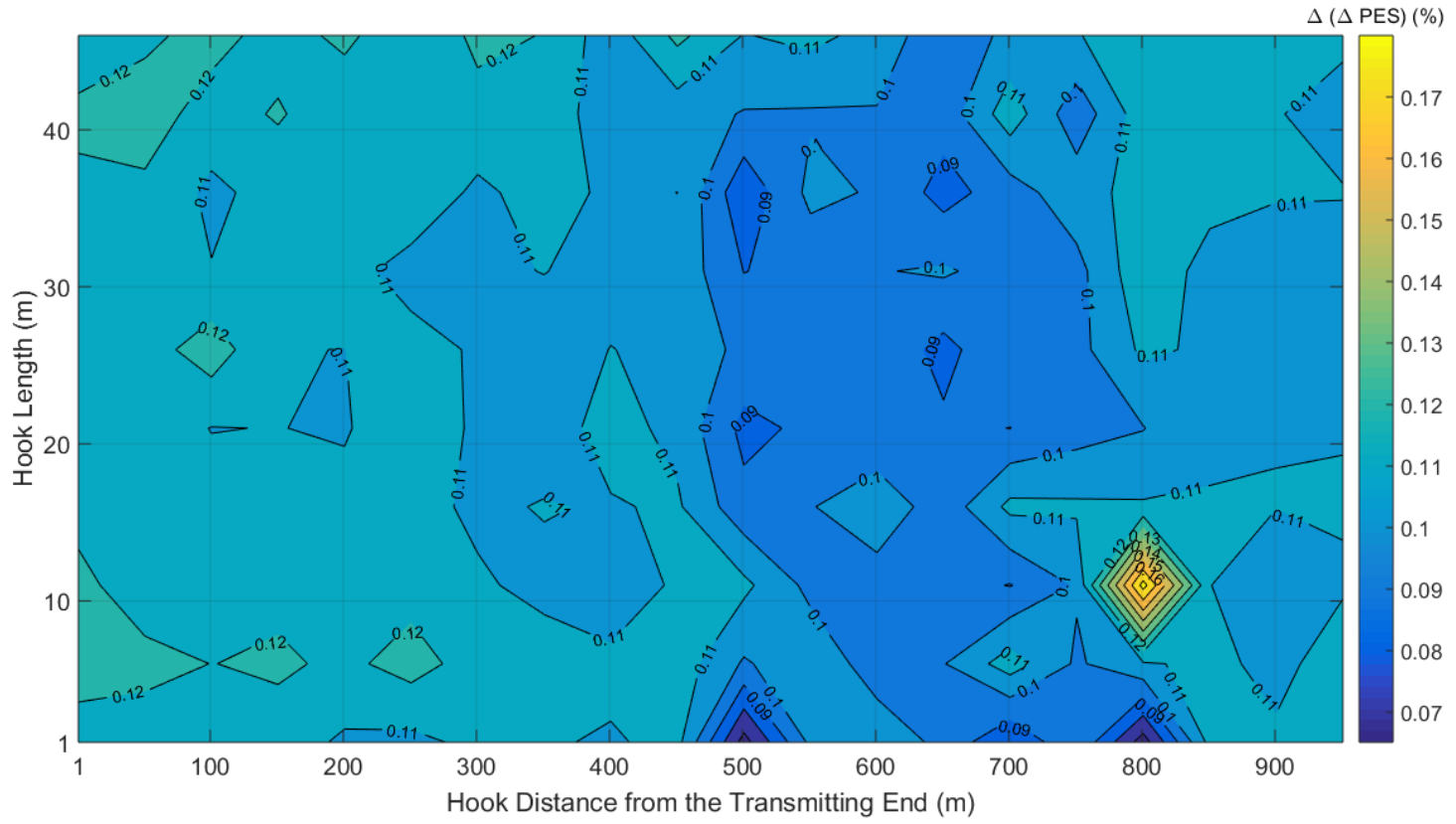

(a)

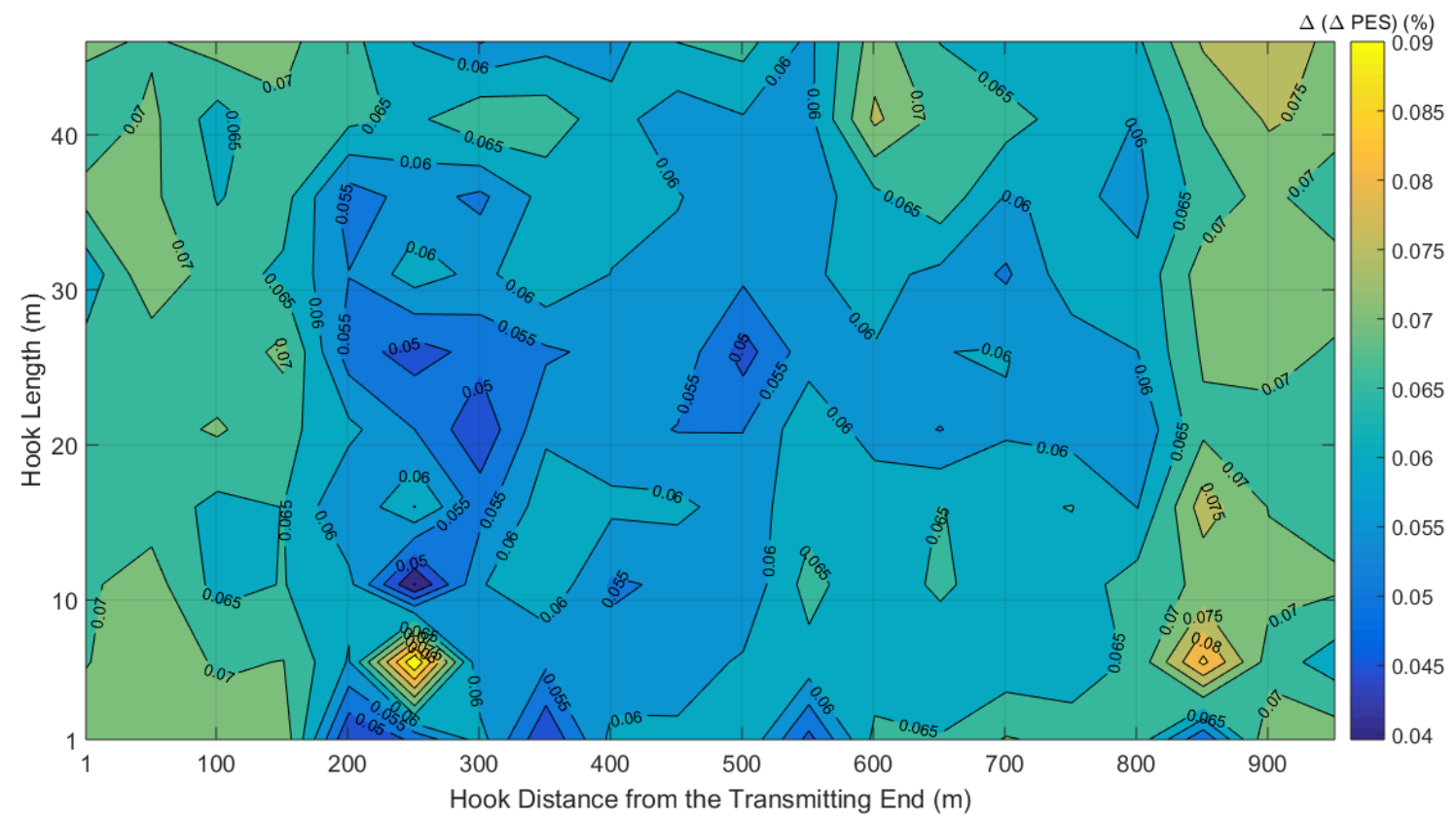

(b) 


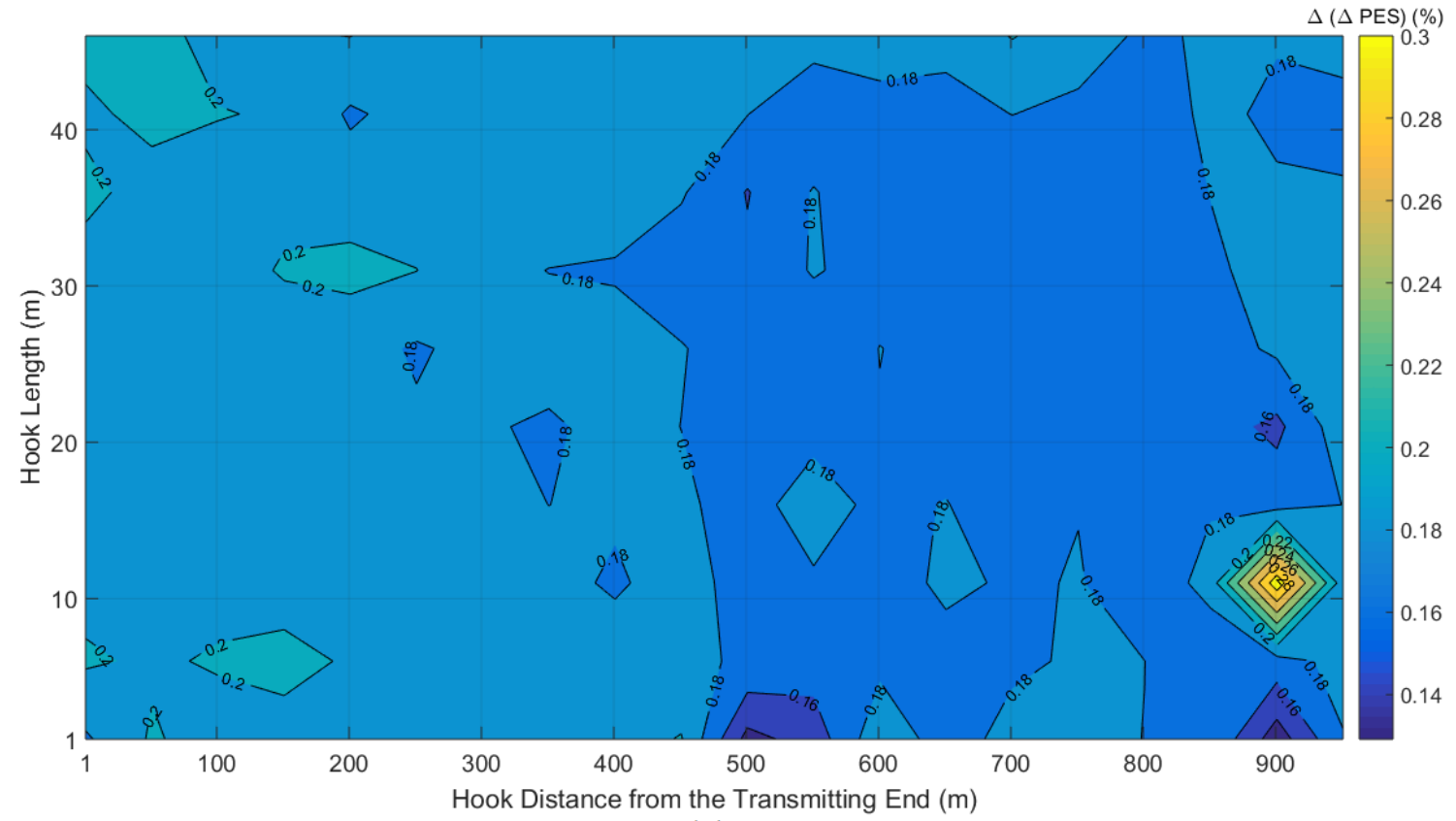

(c)

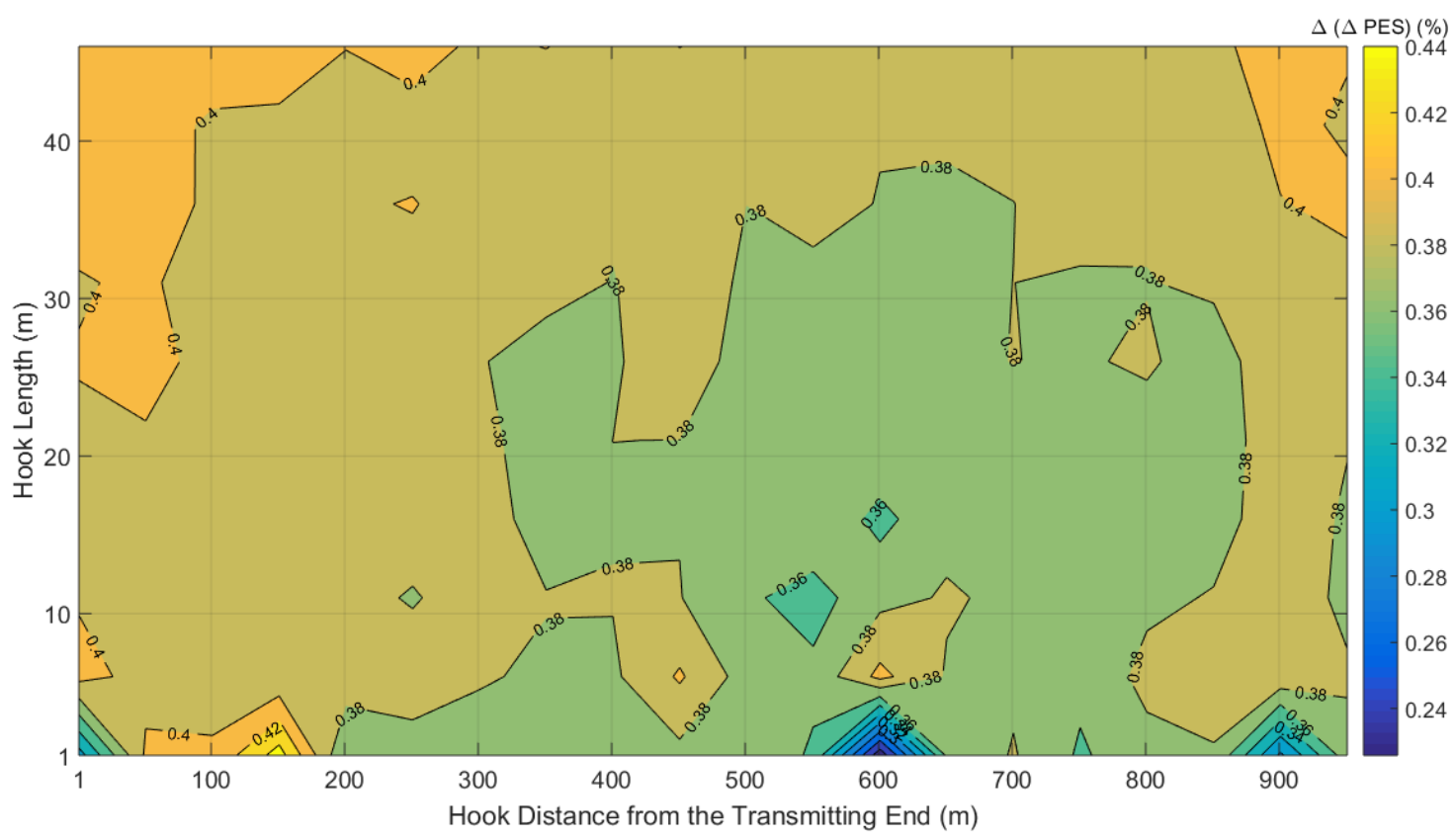

(d) 


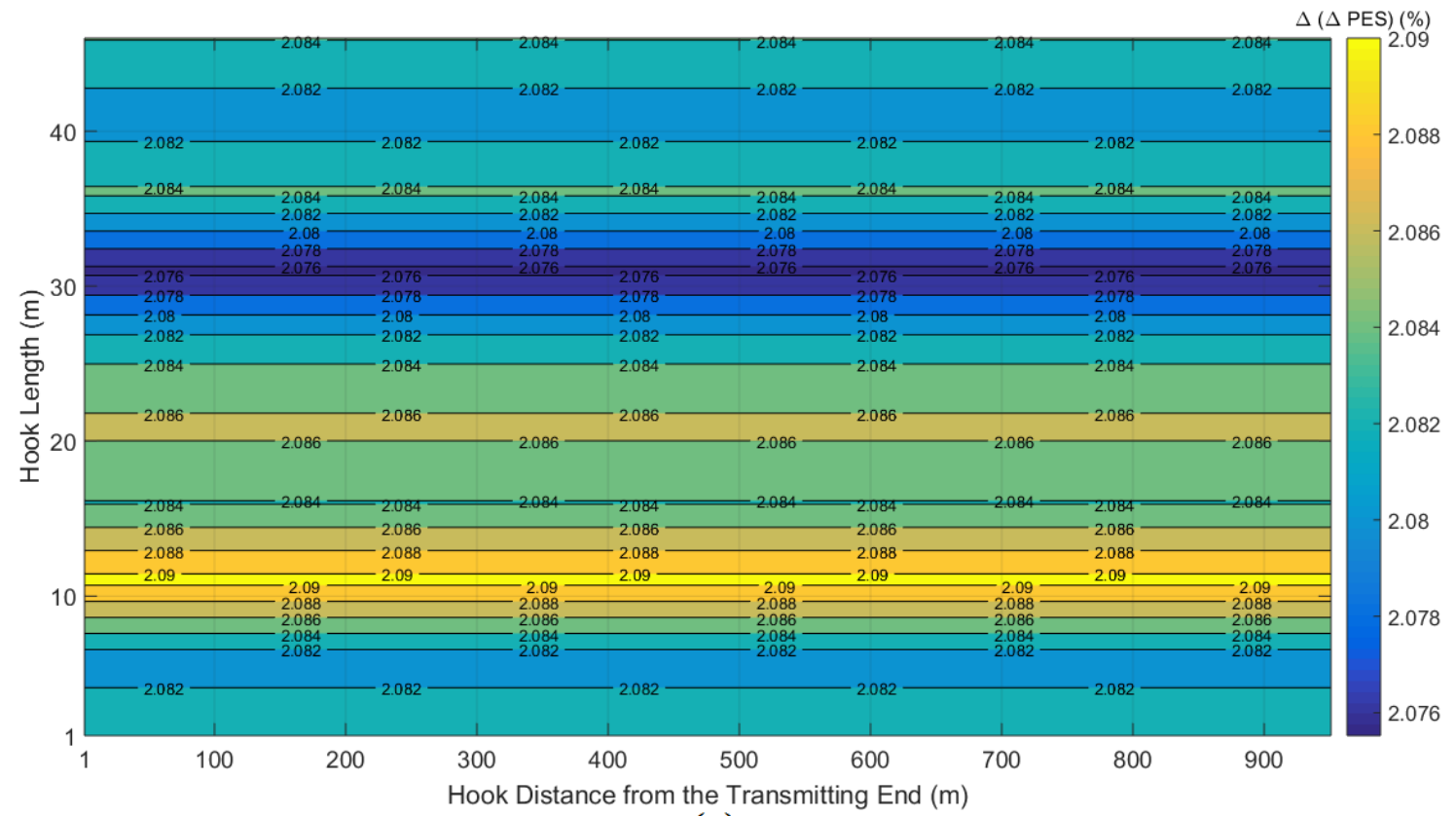

(e)

Fig. 7. Same curves with Fig. 6 but for measurement location deviation $d e v_{\mathrm{t}}$ of $20 \mathrm{~m}$.

the aforementioned areas are less affected by the measurement location deviations of Scenario $\mathrm{B}$ since the lowest in magnitude $\triangle(\triangle P E S)$ values occur there. The opposite situation holds in the areas of more difficult hook style energy theft detection. For example, with reference to Figs. 3(a)-(e) of [20], the hook style energy theft detection in OV LV BPL "LOS" topologies remains easier when short hooks are applied regardless of the distance from the transmitting end while among the lowest $\triangle(\triangle P E S)$ values occur in these cases with reference to Figs. 6(e) and 7(e).

From the previous findings, the main advantage of Scenario B is underlined that is the consideration of longer modified ad-hoc OV LV BPL topologies by the Information Technology department for the hook style energy theft detection since positive $\triangle(\triangle P E S)$ values imply better performance of HS-DET method. When longer modified ad-hoc OV LV BPL topologies are used, the algebraic sum of measurement location deviations $d e v_{\mathrm{t}}$ and $d e v_{\mathrm{r}}$ rather than the exact installation positions of the transmitting and receiving end remain the important issue for Scenario B.

\subsection{HS-DET Method and Longer Ad-Hoc OV LV BPL Topologies than the Typical OV LV BPL Topologies}

When a complete OV LV BPL network is installed, this network can be divided into cascaded OV LV BPL topologies of typical lengths of $1000 \mathrm{~m}$. In contrast, the main advantage of the installation of ad-hoc OV LV BPL topologies can be a more relaxed perspective regarding their lengths. If the hook style energy theft detection remains safe by HS-DET method, significantly longer lengths can be exploited for the ad-hoc OV LV BPL topologies. In order to assess the performance of HS-DET method through $\triangle P E S$, longer ad-hoc OV LV BPL topologies should be examined in urban, 
suburban, rural and "LOS" environments. Note that each environment corresponds to a respective ad-hoc OV LV BPL topology class with reference to Tables 1-3. To examine the length impact of ad-hoc OV LV BPL topologies in the aforementioned environments, concatenated OV LV BPL topologies will be used; for example, the indicative ad-hoc OV LV BPL urban topology of Table 1 is equal to $1000 \mathrm{~m}$, while the respective concatenated ad-hoc OV LV BPL urban topology without hook of $v \times 1000 \mathrm{~m}$ length is given in Table 3 where $v$ is the number of concatenations. Although the concatenation of same OV LV BPL topology classes is a rather simplified and pessimistic approach, it can describe the BPL topology complexity of the urban, suburban, rural and "LOS" environments.

With reference to Tables 1 and 3, let assume that a concatenated ad-hoc OV LV BPL topology of $v$ indicative ad-hoc OV LV BPL topologies (i.e., the number of concatenation is equal to $v$ ) is first installed by the Information Technology department and collect the required reference data. After the uninstallation, a hook style energy theft occurs by using a hook of length $L_{\mathrm{bh}}$ that is hung at distance $D_{\mathrm{h}}$ from the transmitting end. Information Technology department reinstalls the same concatenated ad-hoc OV LV BPL topology without any measurement location deviations. In Fig. 8, $\triangle P E S$ is plotted with respect to the number of concatenation $v$ when $L_{\mathrm{bh}}=5 \mathrm{~m}$ and $D_{\mathrm{h}}=50 \mathrm{~m}$ are assumed for the five concatenated ad-hoc OV LV BPL topologies as described in Table 3. In Fig. 9, same curves with Fig. 8 are given but for $L_{\mathrm{bh}}=40 \mathrm{~m}$ and $D_{\mathrm{h}}=(v \times 1000 / 2+1) \mathrm{m}$ while same curves with Fig. 8 are given in Fig. 10 but for $L_{\mathrm{bh}}=20 \mathrm{~m}$ and $D_{\mathrm{h}}=(v \times 1000-30) \mathrm{m}$.

From Figs. 8-10, interesting findings can be reported concerning the detection of hook style energy theft when longer ad-hoc OV LV BPL topologies are installed by the Information Technology department. More specifically:

- The length and the class of the ad-hoc OV LV BPL topologies play critical role towards the hook style energy theft detection by $\triangle P E S$. However, the adoption of the conservative $1000 \mathrm{~m}$ typical length of OV LV BPL topologies remains an extremely safe decision regardless of the applied ad-hoc OV LV BPL topology class. Anyway, the hook style energy theft detection performance inversely depends on the length of the installed ad-hoc OV LV BPL topology.

- Depending on the examined OV LV BPL topology class, Information Technology department can adaptively install ad-hoc OV LV BPL topologies of different lengths. On the basis of a safe hook style energy theft detection that is assured by the strict $10 \% \triangle P E S$ threshold, Information Technology department can install:

- ad-hoc "LOS" OV LV BPL topologies whose length may exceed $10 \mathrm{~km}$;

- ad-hoc rural OV LV BPL topologies whose length may reach up to $8 \mathrm{~km}$;

$\circ$ ad-hoc suburban OV LV BPL topologies whose length may reach up to 4 $\mathrm{km}$; and

○ ad-hoc urban OV LV BPL topologies whose length may reach up to $2 \mathrm{~km}$ (aggravated cases) even to $3 \mathrm{~km}$ (typical cases).

- Comparing Figs. 8-10, small $\triangle P E S$ differences are observed for given ad-hoc OV LV BPL topology. This is due to the fact the hook length and the hook distance from the transmitting end has smaller effect on $\triangle P E S$ rather than the number of 


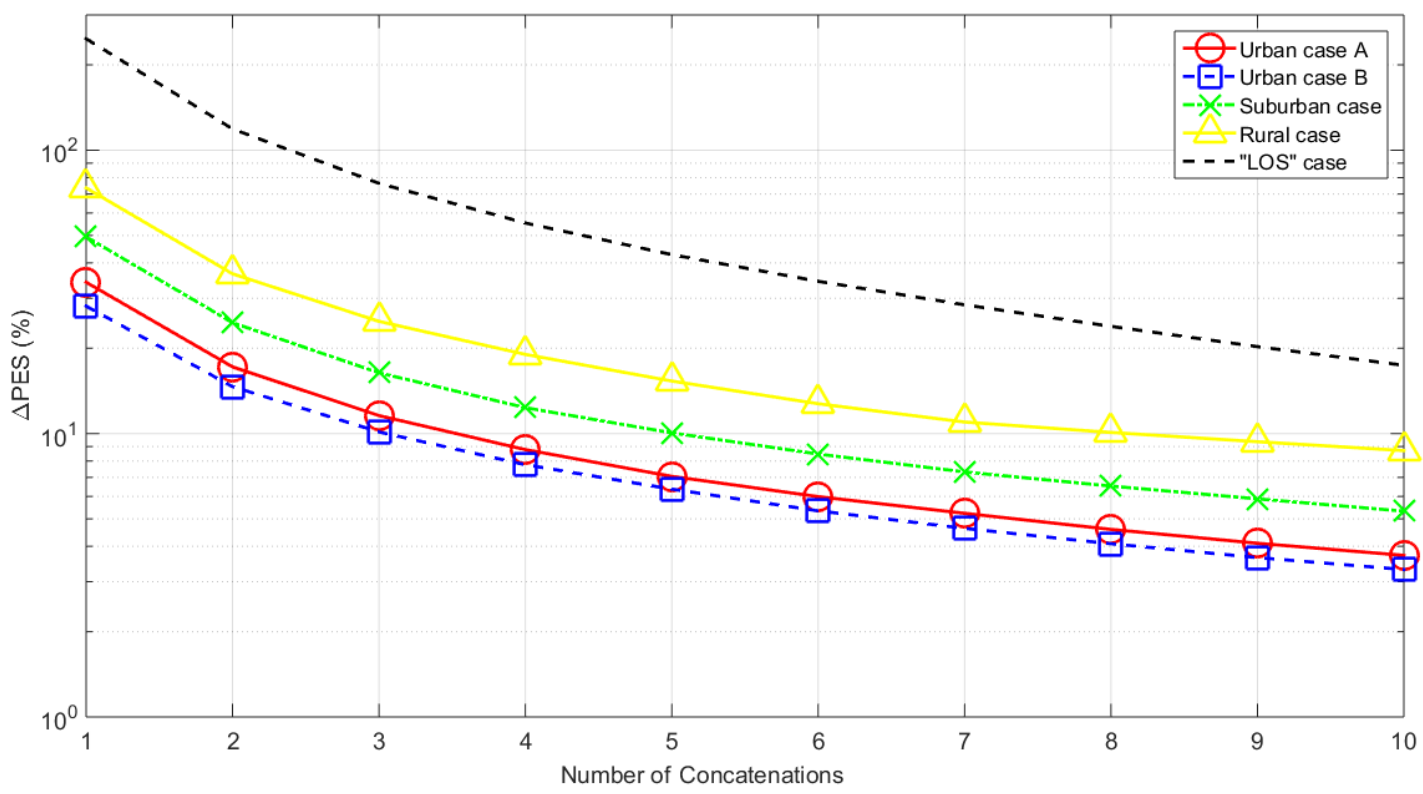

Fig. 8. $\triangle P E S$ of HS-DET method for the five concatenated OV LV BPL topologies of Table 3 when hook length of $5 \mathrm{~m}$ and hook distance from the transmitting end of $50 \mathrm{~m}$ is assumed for different numbers of concatenations ( $y$-axis is in logarithmic scale).

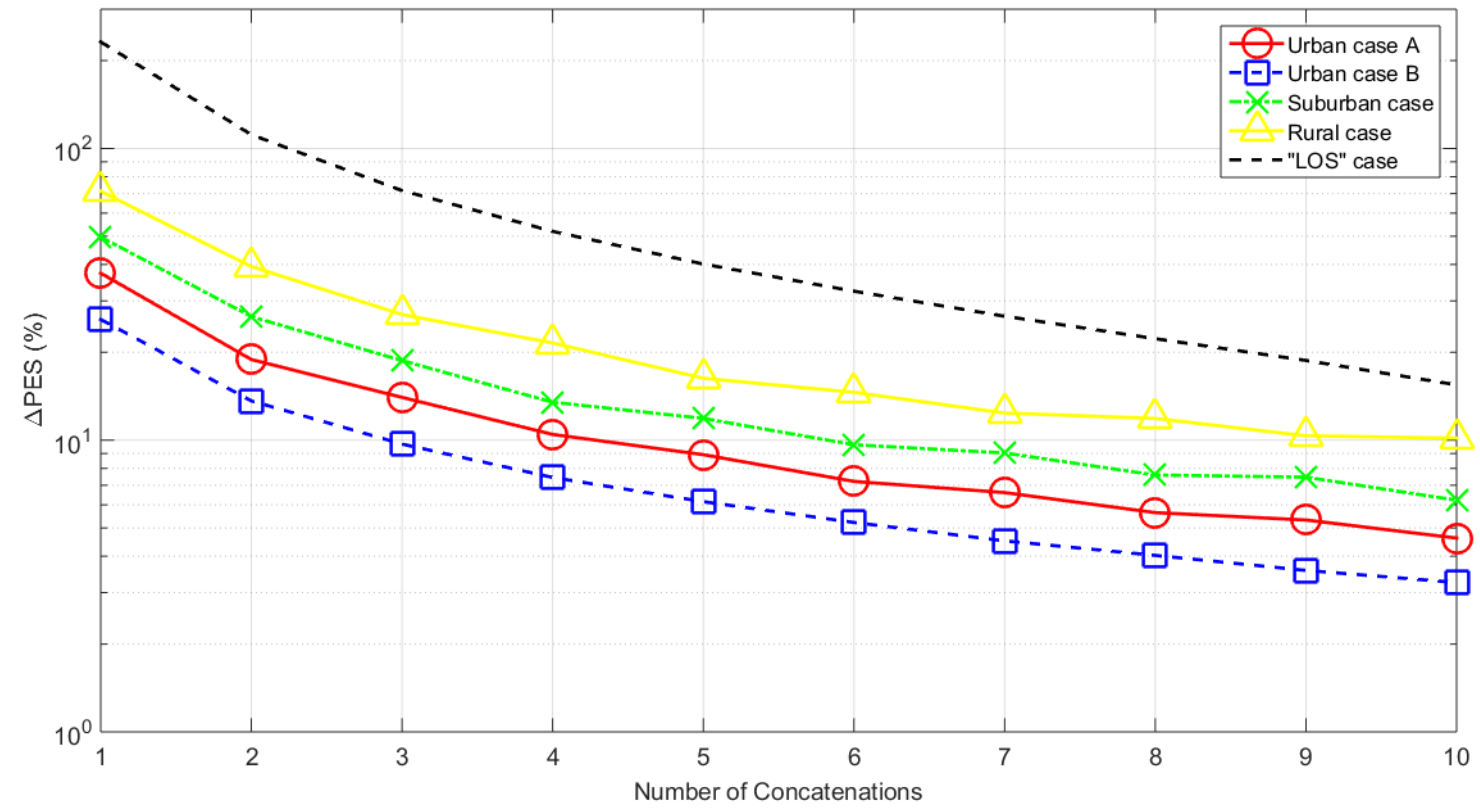

Fig. 9. Same curves with Fig. 8 but for hook length of $40 \mathrm{~m}$ and hook distance from the transmitting end of $(v \times 1000 / 2+1) \mathrm{m}$. 


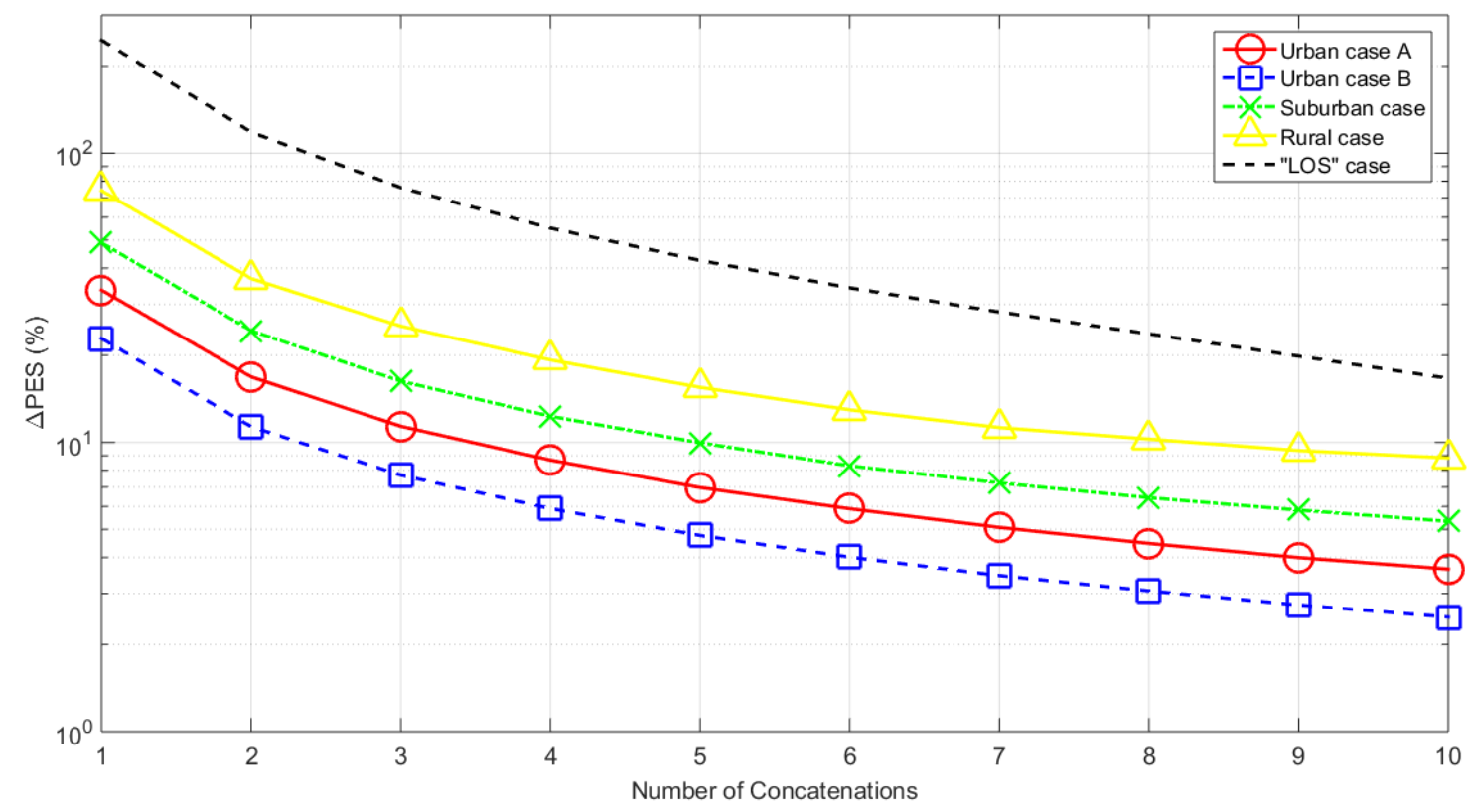

Fig. 10. Same curves with Fig. 8 but for hook length of $20 \mathrm{~m}$ and hook distance from the transmitting end of $(v \times 1000-30) \mathrm{m}$.

concatenations. Anyway, a high number of concatenations implies that stronger multipath environments are created where the channel attenuation imposed by the hook remains smaller than the channel attenuation by the multipath environment because of the $v$ multiple branches.

- In accordance with the findings of Secs. 4.1.1 and 4.1.2, Information Technology department can exploit the longer ad-hoc OV LV BPL topologies even if high accuracy regarding the installation positions lacks. Practically, Information Technology department should carefully report the first and the last branch of the ad-hoc OV LV BPL topology from which the reference measurements had been collected as the effect of additional or fewer MTL cable segments outside the topology range of the first and last branch on $\triangle P E S$ remains negligible.

- In accordance with [20]-[22], measurement differences and various jamming techniques, which are not taken into consideration in this paper, have as impact the reduction of $\triangle P E S$ values. In order to comply with the strict $10 \% \triangle P E S$ threshold, a small reduction of the number of concatenations can counteract this $\triangle P E S$ aggravation.

Concluding this Section, an important remark is that there is no need for deploying a complete OV LV BPL network but a careful installation of an ad-hoc OV LV BPL topology for a hook style energy theft detection. In order to detect a hook style energy theft, longer ad-hoc OV LV BPL topologies than the typical OV LV BPL topologies can be installed by Information Technology department and the hook style energy theft detection can be successfully and easily made even with the strict $10 \% \triangle P E S$ threshold. Successful hook style energy theft detections can be made even if the lengths of the ad-hoc OV LV BPL topologies exceed 10km in "LOS" cases. But among the most critical topics of this Section is that there is no need for absolute 
accuracy regarding the installation of the original and modified ad-hoc OV LV BPL topology. Even if measurement deviations occur at the transmitting and receiving end, the length should be assured by the Information Technology department that remains the same. Even if the length between the original and modified ad-hoc OV LV BPL topology is not the same, the influence of measurement deviations of transmitting and receiving end can be negligible if these measurement deviations are small fractions of the length of the examined ad-hoc OV LV BPL topology.

\section{Conclusions}

This paper has focused on the performance of HS-DET method when ad-hoc OV LV BPL topologies are installed by Information Technology departments of power utilities. In fact, two critical issues regarding the operation of ad-hoc OV LV BPL topologies have been discussed in this paper while their impact on the hook style energy theft detection by $\triangle P E S$ of HS-DET method has been assessed; first, two scenarios regarding the measurement location deviation of the transmitting and receiving ends of the ad-hoc OV LV BPL topologies from the initial reference ad-hoc OV LV BPL topologies have been identified. In Scenario A, it has been proven that small measurement location deviations from the initial positions of the transmitting and receiving ends of the ad-hoc OV LV BPL topologies (i.e., measurement location deviations up to $10 \mathrm{~m}$ ) negligibly affect the hook style energy theft detection due to the symmetry attribute of BPL networks when the length of the original and the shifted ad-hoc OV LV BPL topology remains the same. In Scenario B, the effect of the measurement location deviations from the initial positions of the transmitting and receiving ends of the ad-hoc OV LV BPL topologies has been assessed but without the same length between the original and the shifted ad-hoc OV LV BPL topologies. Again, the hook style energy theft detection practically remains unaffected since $\triangle \mathrm{PES}$ aggravation remains below $1 \%$ in the vast majority of the cases, which is significantly lower than the strict $10 \% \triangle P E S$ threshold for a safe hook style energy theft decision, when small measurement location deviations from the initial positions of the transmitting and receiving ends of the ad-hoc OV LV BPL topologies occur. Second, the impact of longer ad-hoc OV LV BPL topologies on the performance of hook style energy theft detection has been assessed. It has been proven that Information Technology department can deploy ad-hoc OV LV BPL topologies whose length can comfortably exceed $2 \mathrm{~km}$ and even $10 \mathrm{~km}$ depending on the examined OV LV BPL topology class; say, the smart grid environment. In conclusion, a wise management of ad-hoc OV LV BPL topologies by Information Technology department, e.g., an accurate installation plan and an updated database management system, can secure extremely safe decisions concerning the hook style energy theft detection in all the cases examined without the need of additional costs concerning either specialized equipment or a complete BPL network.

\section{CONFLICTS OF INTEREST}

The author declares that there is no conflict of interests regarding the publication of this paper. 


\section{References}

[1] C. H. Lo, and N. Ansari, "CONSUMER: A Novel Hybrid Intrusion Detection System for Distribution Networks in Smart Grid," IEEE Transactions on Emerging Topics in Computing, vol. 1, no. 1, pp. 33-44, 2013.

[2] R. Jiang, R. Lu, Y. Wang, J. Luo, C. Shen, and X. S. Shen, "Energy-theft Detection Issues for Advanced Metering Infrastructure in Smart Grid," Tsinghua Science and Technology, vol. 19, no. 2, pp. 105-120, 2014.

[3] P. Jokar, N. Arianpoo, and V. CM Leung, "Electricity Theft Detection in AMI Using Customers' Consumption Patterns," IEEE Transactions on Smart Grid, vol. 7, no. 1, pp. 216-226, 2016.

[4] S. Salinas, M. Li, and P. Li, "Privacy-preserving Energy Theft Detection in Smart Grids: A P2P Computing Approach," IEEE Journal on Selected Areas in Communications, vol. 31, no. 9, pp. 257-267, 2013.

[5] D. R. Pereira, M. A. Pazoti, L. A. Pereira, D. Rodrigues, C. O. Ramos, A. N. Souza, and J. P. Papa, "Social-Spider Optimization-based Support Vector Machines applied for energy theft detection," Computers \& Electrical Engineering, vol. 49, pp. 25-38, 2016.

[6] S. Salinas, M. Ming, and P. Li, "Privacy-preserving Energy Theft Detection in Smart Grids," Sensor, Mesh and Ad Hoc Communications and Networks (SECON), 2012, in proc. 9th Annual IEEE Communications Society Conference on. IEEE, pp. 605-613, 2012.

[7] S. McLaughlin, B. Holbert, S. Zonouz, and R. Berthier, "AMIDS: A Multi-sensor Energy Theft Detection Framework for Advanced Metering Infrastructures," in Smart Grid Communications (SmartGridComm), in proc of 2012 IEEE Third International Conference on, pp. 354-359, Nov. 2012.

[8] P. McDaniel and S. McLaughlin, "Security and Privacy Challenges in the Smart Grid," IEEE Security \& Privacy, vol. 7, no. 3, pp. 75-77, 2009.

[9] Federal Court of Audit, "Operational Audit Report Held in National Agency of Electrical Energy, ANEEL, Brazil”, Rep. no. TC 025.619/2007-2, Brasília, Brazil, 2007.

[10] Ministry of Power, India, "Overview of power distribution," Tech. Rep., http://www.powermin.nic.in, 2013.

[11] A. G. Lazaropoulos, "Measurement Differences, Faults and Instabilities in Intelligent Energy Systems - Part 1: Identification of Overhead High-Voltage Broadband over Power Lines Network Topologies by Applying Topology Identification Methodology (TIM)," Trends in Renewable Energy, vol. 2, no. 3, pp. $85-112$, Oct. 2016.

[12] A. G. Lazaropoulos, "Measurement Differences, Faults and Instabilities in Intelligent Energy Systems - Part 2: Fault and Instability Prediction in Overhead High-Voltage Broadband over Power Lines Networks by Applying Fault and Instability Identification Methodology (FIIM)," Trends in Renewable Energy, vol. 2, no. 3, pp. 113 - 142, Oct. 2016.

[13] A. G. Lazaropoulos, "Power Systems Stability through Piecewise Monotonic Data Approximations - Part 2: Adaptive Number of Monotonic Sections and Performance of L1PMA, L2WPMA and L2CXCV in Overhead Medium-Voltage Broadband over Power Lines Networks," Trends in Renewable Energy, vol. 3, no. 1, pp. 33 -60, Jan. 2017. 
[14] A. G. Lazaropoulos, "Power Systems Stability through Piecewise Monotonic Data Approximations - Part 1: Comparative Benchmarking of L1PMA, L2WPMA and L2CXCV in Overhead Medium-Voltage Broadband over Power Lines Networks," Trends in Renewable Energy, vol. 3, no. 1, pp. 2 - 32, Jan. 2017.

[15] A. N. Stefanidi, I. A. Panos, A. N. Milioudis, and G. T. Andreou, "Sympathetic Tripping in a Field Case Study," In 2018 IEEE 53rd International Universities Power Engineering Conference (UPEC), pp. 1-5, Sep. 2018.

[16] A. G. Paspatis, G. C. Konstantopoulos, T. A. Papadopoulos, and V. C. Nikolaidis, "Dynamic grid voltage support from distributed energy resources during shortcircuits," In 2017 IEEE 52nd International Universities Power Engineering Conference (UPEC), pp. 1-6, Aug. 2017.

[17] A. G. Lazaropoulos, "Main Line Fault Localization Methodology in Smart Grid Part 1: Extended TM2 Method for the Overhead Medium-Voltage Broadband over Power Lines Networks Case," Trends in Renewable Energy, vol. 3, no. 3, pp. 2-25, Dec. 2017.

[18] A. G. Lazaropoulos, "Main Line Fault Localization Methodology in Smart Grid Part 2: Extended TM2 Method, Measurement Differences and L1 Piecewise Monotonic Data Approximation for the Overhead Medium-Voltage Broadband over Power Lines Networks Case," Trends in Renewable Energy, vol. 3, no. 3, pp. 26-61, Dec. 2017.

[19] A. G. Lazaropoulos, "Main Line Fault Localization Methodology in Smart Grid Part 3: Main Line Fault Localization Methodology (MLFLM)," Trends in Renewable Energy, vol. 3, no. 3, pp. 62-81, Dec. 2017.

[20] A. G. Lazaropoulos, "Detection of Energy Theft in Overhead Low-Voltage Power Grids - The Hook Style Energy Theft in the Smart Grid Era," Trends in Renewable Energy, vol. 5, no. 1, pp. 12 - 46, Oct. 2018.

[21] A. G. Lazaropoulos, "Special Cases during the Detection of the Hook Style Energy Theft in Overhead Low-Voltage Power Grids through HS-DET Method - Part 1: High Measurement Differences, Very Long Hook Technique and "Smart" Hooks," Trends in Renewable Energy, vol. 5, no. 1, pp. 60-89, Jan. 2019.

[22] A. G. Lazaropoulos, "Special Cases during the Detection of the Hook Style Energy Theft in Overhead Low-Voltage Power Grids through HS-DET Method - Part 2: Different Measurement Differences, Feint "Smart" Hooks and Hook Interconnection Issues," Trends in Renewable Energy, vol. 5, no. 1, pp. 90-116, Jan. 2019.

[23] A. G. Lazaropoulos, "Towards Modal Integration of Overhead and Underground Low-Voltage and Medium-Voltage Power Line Communication Channels in the Smart Grid Landscape: Model Expansion, Broadband Signal Transmission Characteristics, and Statistical Performance Metrics (Invited Paper)," ISRN Signal Processing, vol. 2012, Article ID 121628, pp. 1-17, 2012. [Online]. Available: http://www.hindawi.com/isrn/sp/2012/121628/

[24] A. G. Lazaropoulos, "Towards broadband over power lines systems integration: Transmission characteristics of underground low-voltage distribution power lines," Progress in Electromagnetics Research B, 39, pp. 89-114, 2012. [Online]. Available: http://www.jpier.org/PIERB/pierb39/05.12012409.pdf

[25] A. G. Lazaropoulos and P. G. Cottis, "Transmission characteristics of overhead medium voltage power line communication channels," IEEE Trans. Power Del., vol. 24, no. 3, pp. 1164-1173, Jul. 2009. 
[26] A. G. Lazaropoulos and P. G. Cottis, "Capacity of overhead medium voltage power line communication channels," IEEE Trans. Power Del., vol. 25, no. 2, pp. 723733, Apr. 2010.

[27] A. G. Lazaropoulos and P. G. Cottis, "Broadband transmission via underground medium-voltage power lines-Part I: transmission characteristics," IEEE Trans. Power Del., vol. 25, no. 4, pp. 2414-2424, Oct. 2010.

[28] A. G. Lazaropoulos and P. G. Cottis, "Broadband transmission via underground medium-voltage power lines-Part II: capacity," IEEE Trans. Power Del., vol. 25, no. 4, pp. 2425-2434, Oct. 2010.

[29] A. G. Lazaropoulos, "Broadband transmission characteristics of overhead highvoltage power line communication channels," Progress in Electromagnetics Research B, vol. 36, pp. 373-398, 2012. [Online]. Available: http://www.jpier.org/PIERB/pierb36/19.11091408.pdf

[30] A. G. Lazaropoulos, "Green Overhead and Underground Multiple-Input MultipleOutput Medium Voltage Broadband over Power Lines Networks: Energy-Efficient Power Control," Springer Journal of Global Optimization, vol. 2012 / Print ISSN 0925-5001, pp. 1-28, Oct. 2012.

[31] A. G. Lazaropoulos, "Deployment Concepts for Overhead High Voltage Broadband over Power Lines Connections with Two-Hop Repeater System: Capacity Countermeasures against Aggravated Topologies and High Noise Environments," Progress in Electromagnetics Research B, vol. 44, pp. 283-307, 2012. [Online]. Available: http://www.jpier.org/PIERB/pierb44/13.12081104.pdf

[32] A. G. Lazaropoulos, "Broadband transmission and statistical performance properties of overhead high-voltage transmission networks," Hindawi Journal of Computer Networks and Commun., 2012, article ID 875632, 2012. [Online]. Available: http://www.hindawi.com/journals/jcnc/aip/875632/

[33] P. Amirshahi and M. Kavehrad, "High-frequency characteristics of overhead multiconductor power lines for broadband communications," IEEE J. Sel. Areas Commun., vol. 24, no. 7, pp. 1292-1303, Jul. 2006.

[34] T. Calliacoudas and F. Issa, "“Multiconductor transmission lines and cables solver," An efficient simulation tool for plc channel networks development," presented at the IEEE Int. Conf. Power Line Communications and Its Applications, Athens, Greece, Mar. 2002.

[35] T. Sartenaer and P. Delogne, "Deterministic modelling of the (Shielded) outdoor powerline channel based on the multiconductor transmission line equations," IEEE J. Sel. Areas Commun., vol. 24, no. 7, pp. 1277-1291, Jul. 2006.

[36] C. R. Paul, Analysis of Multiconductor Transmission Lines. New York: Wiley, 1994.

[37] H. Meng, S. Chen, Y. L. Guan, C. L. Law, P. L. So, E. Gunawan, and T. T. Lie, "Modeling of transfer characteristics for the broadband power line communication channel," IEEE Trans. Power Del., vol. 19, no. 3, pp. 1057-1064, Jul. 2004.

[38] B. Li, D. Mansson, and G. Yang, "An efficient method for solving frequency responses of power-line networks," Progress In Electromagnetics Research B, Vol. 62, 303-317, 2015. doi:10.2528/PIERB15013008 http://www.jpier.org/pierb/pier.php?paper $=15013008$

[39] M. Chaaban, K. El KhamlichiDrissi, and D. Poljak, "Analytical model for electromagnetic radiation by bare-wire structures," Progress In Electromagnetics 
Research B, Vol. 45, 395-413, 2012. doi:10.2528/PIERB12091102 http://www.jpier.org/pierb/pier.php?paper=12091102

[40] Y. H. Kim, S. Choi, S. C. Kim, and J. H. Lee, "Capacity of OFDM two-hop relaying systems for medium-voltage power-line access networks," IEEE Trans. Power Del., vol. 27, no. 2, pp. 886-894, Apr. 2012.

[41] P. Amirshahi and M. Kavehrad, "Medium voltage overhead powerline broadband communications; Transmission capacity and electromagnetic interference," in Proc. IEEE Int. Symp. Power Line Commun. Appl., Vancouver, BC, Canada, Apr. 2005, pp. 2-6.

[42] M. Tang, and M. Zhai, "Research of transmission parameters of four-conductor cables for power line communication," in Proc. Int. Conf. on Computer Science and Software Engineering, Wuhan, China, Dec. 2008, vol. 5, pp. 1306-1309.

[43] M. D'Amore and M. S. Sarto, "A new formulation of lossy ground return parameters for transient analysis of multiconductor dissipative lines," IEEE Trans. Power Del., vol. 12, no. 1, pp. 303-314, Jan. 1997.

[44] M. D'Amore and M. S. Sarto, "Simulation models of a dissipative transmission line above a lossy ground for a wide-frequency range-Part I: Single conductor configuration," IEEE Trans. Electromagn. Compat., vol. 38, no. 2, pp. 127-138, May 1996.

[45] M. D'Amore and M. S. Sarto, "Simulation models of a dissipative transmission line above a lossy ground for a wide-frequency range-Part II: Multi-conductor configuration," IEEE Trans. Electromagn. Compat., vol. 38, no. 2, pp. 139-149, May 1996.

[46] N. Theethayi, "Electromagnetic interference in distributed outdoor electrical systems, with an emphasis on lightning interaction with electrified railway network," Ph.D. dissertation, Uppsala Univ., Uppsala, Sweden, Sep. 2005, [Online]. Available:

portal.org/smash/get/diva2:166746/FULLTEXT01

[47] A. G. Lazaropoulos, "Review and Progress towards the Common Broadband Management of High-Voltage Transmission Grids: Model Expansion and Comparative Modal Analysis," ISRN Electronics, vol. 2012, Article ID 935286, pp. 1-18, 2012. [Online]. Available: http://www.hindawi.com/isrn/electronics/2012/935286/

[48] A. G. Lazaropoulos, "New Coupling Schemes for Distribution Broadband over Power Lines (BPL) Networks," Progress in Electromagnetics Research B, vol. 71, pp. 39-54, 2016. [Online]. Available: http://www.jpier.org/PIERB/pierb71/02.16081503.pdf

[49] A. G. Lazaropoulos, "Broadband Performance Metrics and Regression Approximations of the New Coupling Schemes for Distribution Broadband over Power Lines (BPL) Networks," Trends in Renewable Energy, vol. 4, no. 1, pp. 43 73, 2018.

[50] T. C. Banwell and S. Galli, "On the symmetry of the power line channel," in Proc. Int. Symp. Power-Lines Commun, pp. 325-330. 2001.

[51] S. Galli and T. Banwell, "A novel approach to the modeling of the indoor power line channel-Part II: Transfer function and its properties," IEEE Transactions on Power Delivery, vol. 20, no. 3, pp. 1869-1878, 2005. 
Article copyright: (C) 2019 Athanasios G. Lazaropoulos. This is an open access article distributed under the terms of the Creative Commons Attribution 4.0 International License, which permits unrestricted use and distribution provided the original author and source are credited. 